\title{
Belastung des Neusiedler Sees mit anthropogenen Spurenstoffen: Überlegungen zu Herkunft und Verhalten
}

\author{
Matthias Zessner (iD - Ottavia Zoboli • Daniela Reif · Arabel Amann · Elisabeth Sigmund • Georg Kum • \\ Zdravka Saracevic · Ernis Saracevic · Steffen Kittlaus · Jörg Krampe · Georg Wolfram
}

Zusammenfassung Im vorliegenden Artikel wird auf Basis unterschiedlicher Datenquellen in einem ersten Schritt die Belastung des Neusiedler Sees mit national oder gemeinschaftlich geregelten anthropogenen Spurenstoffen dargestellt. Darüber hinaus werden für ausgewählte Stoffe Eintragspfade in Wulka und Neusiedler See identifiziert und für weitere Stoffe wird ein mögliches Umweltverhalten im See dargestellt. Auch wenn derzeit ein umfassendes Monitoring von allen national oder gemeinschaftlich geregelten Spurenstoffen im Neusiedler See fehlt, kann für den überwiegenden Teil dieser Stoffe auf Basis von Untersuchungen in der Wulka bzw. Fischuntersuchungen im See eine Einhaltung der UQN angenommen werden. Für einzelne Stoffe ist eine Zielverfehlung geben oder anzunehmen. Für andere Stoffe ist zurzeit aufgrund analytischer Schwierigkeiten ein eindeutiger Befund nicht möglich. In Abhängigkeit vom Stoff und der Betrachtungsebene können sowohl Kläranlagenabläufe, landwirtschaftliche Erosion als auch die atmosphärische Deposition auf die Oberfläche des Sees die Einträge dominieren. Mit Bedachtnahme auf die große Zahl der in die aquatische Umwelt emittier-

ao.Univ.-Prof. DI Dr. M. Zessner $(\varangle)$. Dr. O. Zoboli, MSc · DI D. Reif, MSc .

DI A. Amann, BSc · DI Z. Saracevic .

DI Dr. E. Saracevic .

Dipl.-Geoökol. S. Kittlaus .

Univ.-Prof. Dr.-Ing. J. Krampe

Institut für Wassergüte und

Ressourcenmanagement,

Technische Universität Wien,

Karlsplatz 13, A-1040 Wien, Österreich

mzessner@iwag.tuwien.ac.at

Mag. E. Sigmund, MSc .

Mag. MAS G. Kum .

Mag. Dr. G. Wolfram

DWS Hydro-Ökologie,

Zentagasse 47, A-1050 Wien,

Österreich ten anthropogenen Spurenstoffe muss unabhängig von der Betrachtung von Einzelstoffen für den Neusiedler See eine ganz besondere Vulnerabilität für diese Form der Belastung diagnostiziert werden. Dies liegt vor allem an der Funktion des Sees als Stoffsenke. Persistente Spurenstoffe, die in den See gelangen, werden im Freiwasser aufkonzentriert oder in den Sedimentbereichen des Schilfs gespeichert, von wo sie allenfalls wieder mobilisiert werden können. Selbst wenn eine Vielzahl von schwer abbaubaren Spurenstoffen durch Ab- oder Umbau aus dem Freiwasser des Sees eliminiert wird, ist über die Endprodukte eines solchen Umbaus noch wenig bekannt bzw. sind diese aufgrund der Vielzahl möglicher Stoffe nicht gänzlich zu überwachen. Als Grundlage zur Entwicklung von Strategien für ein langfristiges und effizientes Management des Sees wäre ein regelmäßiges Monitoring von Spurenstoffen in See und Schilfgürtel deutlich zu verstärken, um Fehlentwicklungen im See frühzeitig erkennen zu können.

\section{Schlüsselwörter}

Flusseinzugsgebietsmanagement .

Persistenz - Schilfsediment .

Synthetische Chemikalien .

Umweltmonitoring

Umweltqualitätsnormen

\section{Contamination of Lake Neusiedl with anthropogenic trace substances: considerations on their origin and behaviour}

Abstract In this paper, we first present the contamination of Lake Neusiedl with anthropogenic trace substances regulated at national or EU level. Second, we identify main emission pathways for selected substances into the River Wulka and Lake Neusiedl and identify the potential environmental behaviour of trace substances in the aquatic system. Even if a comprehensive monitoring of all regulated substances in the lake is at present still missing, we can assume the compliance with environmental quality standards for most of them based on measurements carried out in the River Wulka and from biota-monitoring in the lake. For some substances quality criteria are not or probably not met, for others a final diagnosis is currently not possible due to analytical constraints. Depending on the examined substance, effluents from waste water treatment plants, agricultural erosion or atmospheric deposition on the lake surface may be the dominant pathway of contamination into the River Wulka and Lake Neusiedl. Besides specific considerations for individual substances, taking into account the enormous number of anthropogenic trace substances that are released into the environment, Lake Neusiedl must be regarded as especially vulnerable to this kind of contamination. The high vulnerability mainly derives from the lake acting as a substance-sink. Persistent chemicals which enter the lake may concentrate in the water of the lake or may be stored in the sediments of the reed belt, from where they might be mobilized later on. Even if many of the highly persistent chemicals are removed from the lake water through degradation or conversion to metabolites, little is known about the end products of this conversion and they cannot be monitored completely because of their high number. As a basis for the development of strategies for a long-term and efficient management of the lake, a regular monitoring of trace substances in lake and reed belt should be reinforced considerably to detect any undesirable developments as early as possible.

Keywords Environmental monitoring . Environmental quality standards . Persistence $\cdot$ Reed belt sediment $\cdot$ River basin management $\cdot$ Synthetic chemicals 


\section{Einleitung}

Anthropogene Spurenstoffe wird die große Gruppe von synthetischen Chemikalien genannt, die durch unterschiedliche Nutzungen in die Umwelt gelangen, dort in sehr geringen Konzentrationen vorkommen, aber auch bei geringen Konzentrationen kritische Wirkungen an Lebewesen in Gewässern hervorrufen können (ÖWAV 2013). Aus diesem Grund wird diese Stoffgruppe im allgemeinen Sprachgebrauch auch organische Mikroverunreinigungen, Schadstoffe oder gefährliche Stoffe genannt. In der Europäischen Union gibt es eine Vielzahl von Vorgaben, die bereits den Einsatz dieser Stoffe regeln (z.B. REACH, EU 2006). Für die aquatische Umwelt wurden im Zuge der Wasserrahmenrichtlinie (EU 2000) von Seiten der EU prioritäre und prioritär gefährliche Stoffe definiert und Umweltqualitätsnormen (UQN) festgelegt, welche in den Mitgliedstaaten verbindlich $\mathrm{zu}$ einzuhalten sind (EU 2008/2013). Neben 4 Metallen sind es 54 anthropogene Spurenstoffe, die zu den EU-weit geregelten prioritären Stoffen zählen. Darüber hinaus wurden in der österreichischen Qualitätszielverordnung Chemie Oberflächengewässer (QZV Chemie OG, BGBl II Nr. 96/2006 idgF) für 34 weitere Stoffe (davon 24 anthropogene, organische Spurenstoffe) UQN festgelegt.
Aus der großen Gruppe der anthropogenen Spurenstoffe stellen die auf EU-Ebene und national in der QZV Chemie OG geregelten Stoffe nur einen kleinen Ausschnitt dar. Damit geht auch das Problem einher, die Liste der geregelten Stoffe aktuell zu halten. Stoffe, bei denen auf EU-Ebene eine Zuordnung $\mathrm{zu}$ den prioritären Stoffen überlegt wird, werden in eine Beobachtungsliste aufgenommen (EU 2008/2013). Weitere Untersuchungen auf Basis von Mess- oder Modellierungsergebnissen geben Priorisierungen vor, um aus einer Vielzahl von Stoffen (bis zu 1800) jene Stoffe und Stoffgruppen $\mathrm{zu}$ identifizieren, denen in Zukunft teilweise auch einzugsgebietsspezifisch vermehrt Beachtung geschenkt werden sollte (Carvalho et al. 2016; Slobotnik und von der Ohe 2015; van Gils et al. 2019). Die riesige Anzahl von Stoffen (derzeit wurden bereits mehr als 130 Mio. Stoffe registriert, Abb. 1; CAS 2018) stellt auch eine große Herausforderung für die Zukunft des Gewässerschutzes dar. Konzepte werden sich zum einen auf Wirkungsanalysen mit Summenparametern und zum anderen auf ausgewählte Indikatorsubstanzen stützen, die neben einer ökotoxikologischen Relevanz aufgrund ihrer Einsatzweise und ihres Umweltverhaltens stellvertretend für viele Stoffe betrachtet werden können. Die Planung von Maßnahmen erfordert dann einen stoff(gruppen)spezifischen und auch die charakteristischen Nutzungen und die Rahmenbedingungen spezifischer Einzugsgebiete berücksichtigenden Ansatz (Fenz und Clara 2019).

Eine wirkungsvolle Verringerung der Gesamtbelastung der Gewässer ist wahrscheinlich nicht durch isolierte Maßnahmen zu erreichen, sondern durch Kombinationen von Maßnahmen, die den gesamten Lebenszyklus von Stoffen berücksichtigen. Diesem Ansatz folgen die Empfehlungen der österreichischen Wasserwirtschaft im Positionspapier „Anthropogene Spurenstoffe" (ÖWAV 2013), die Empfehlungen des deutschen Umweltbundesamtes zur Reduzierung von Mikroverunreinigungen in den Gewässern (UBA 2018) als auch die Ansätze zur "circular economy“ auf europäischer Ebene (Fenz und Clara 2019). Grundlage entsprechender Ansätze ist ein gutes Verständnis der Herkunftsmuster und des Umweltverhaltens der Chemikalien unter spezifischen regionalen Rahmenbedingungen. Dieses zu erarbeiten ist eine herausfordernde Aufgabe für die wasserwirtschaftliche Forschung.

Der Neusiedler See hat nicht nur eine Reihe von anderen gesellschaftlich und ökologisch wichtigen Funktionen, sondern stellt auch eine wichtige Stoffsenke dar. Stoffe werden über Zuflüsse oder atmosphärische Deposition in den See eingetragen. Aufgrund der Domi-

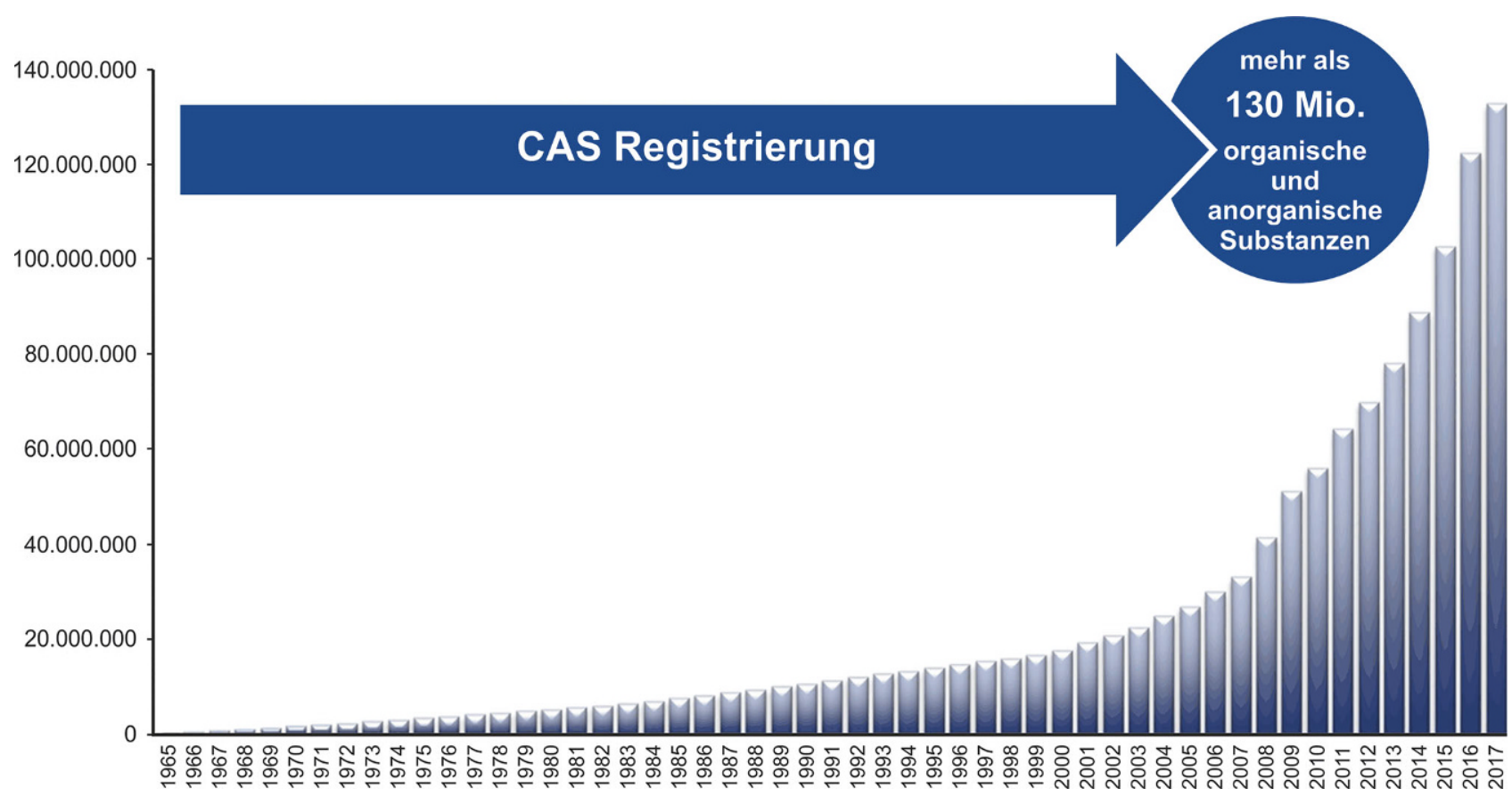

Abb. 1 Entwicklung der Anzahl von beim Chemical Abstracts Service (CAS) registrierten Chemikalien (modifiziert nach CAS 2018) 
nanz der Verdunstung gegenüber dem Niederschlag bzw. des Zuflusses gegenüber dem Abfluss hat der See, zusätzlich zu seiner Funktion als Absetzbecken für Feststoffe, ein Potenzial zu einer Anreicherung von gelösten Stoffen. Dies kann einerseits zu einer Konzentrationszunahme in der flüssigen Phase führen oder über eine Adsorption an Schwebstoffe und deren Sedimentation zu einer Verlagerung und Speicherung im Schilfgürtel, ein Prozess, dessen langfristige Auswirkungen weitgehend unbekannt sind. Andererseits sind die Aufenthaltszeiten von Wasser und Stoffen im See sowie - aufgrund der geringen Tiefe - die UV-Bestrahlung relativ hoch. Dadurch ergeben sich Bedingungen, die einen biologischen, vor allem aber einen photokatalytischen Ab- oder Umbau organischer Stoffe gegenüber anderen aquatischen Ökosystemen vergleichsweise begünstigen. Während die Belastung des Sees mit Nährstoffen oder Summenparametern für die organische Substanz (TOC, DOC) schon seit langem ein wichtiger Untersuchungsgegenstand ist und damit ein hoher Wissenstand gegeben ist (z.B. Stalzer und Spatzierer 1987; Wolfram und Herzig 2013; Wolfram et al. 2019) sowie gezielte Untersuchungen $\mathrm{zu}$ einzelnen Schwermetallen vorliegen (z. B. Jirsa et al. 2014), wurde die Belastung des Neusiedler Sees mit anthropogenen Spurenstoffen bisher erst wenig untersucht (Wolfram et al. 2014). Ziel dieses Artikels ist es daher, eine Standortbestimmung in Hinblick auf die aktuelle Belastungssituation sowie zur Herkunft und dem Verhalten anthropogener Spurenstoffe im Neusiedler See zu versuchen.

\section{Aktuelle Belastungssituation}

\subsection{Datenlage}

Für gemeinschaftsrechtlich oder national geregelte Stoffe ist es Aufgabe der nationalen Verwaltung im Zuge der Umsetzung der WRRL, die Einhaltung der UQN in den österreichischen Gewässern zu überwachen. UQN werden in Abhängigkeit vom jeweiligen Parameter als Jahresdurchschnitts-Umweltqualitätsnorm (JD-UQN), als ZulässigeHöchstkonzentrations-Umweltqualitätsnorm (ZHK-UQN) oder als Umweltqualitätsnorm in Biota (Konzentrationen in Fischen bzw. Krebsen und Weichtieren bezogen auf die Feuchtsubstanz) (Biota-UQN) vorgegeben
(BGBl II Nr. 96/2006 idgF). Die Anforderungen an die Zustandsüberwachung sind in der Gewässerzustandsüberwachungsverordnung (GZÜV, BGBl. II Nr. 479/2006, idgF) geregelt.

Im Neusiedler See wurden im Zuge eines nationalen Fischmonitoringprogramms im Jahr 2013 die Biota-Gehalte organischer Spurenstoffe gemessen (Clara et al. 2015). Im Monitoringprogramm waren folgende Stoffe enthalten, für die Biota-UQN festgelegt sind: bromierte Diphenylether (PBDE, Summe der Kongenere der Nummern 28, 47, 99, 100, 153 und 154), Hexachlorbenzol, Hexachlorbutadien, Dicofol, Perfluoroktansulfonsäure (PFOS), Dioxine und dioxinähnliche Verbindungen (PCDD+PCDF+PCB-DL), Hexabromcyclododecan (HBCDD), Heptachlor und Heptachlorepoxid. Von Seiten der Metalle besteht für Quecksilber eine BiotaUQN. Quecksilber war daher ebenfalls Teil dieses Fischmonitorings. Aus den Stoffen mit Biota-UQN wurden Fluoranthen und Benzo(a)pyren aus der Gruppe der polyzyklischen aromatischen Kohlenwasserstoffe (PAK) nicht analysiert, weil diese Stoffe für die $\mathrm{Zu}$ standsbewertung bisher im Wasser untersucht wurden und das Trendmonitoring im Sediment durchgeführt wurde. Die Biota-UQN ist zudem auf Krebsund Schalentiere bezogen, die im Rahmen des Fischmonitoringprogramms aus 2013 nicht untersucht wurden.

Für bioakkumulierende Stoffe hat eine Erfassung des Gewässerzustandes über Biota-Untersuchungen den Vorteil einer höheren Sensitivität der Untersuchungen durch die Stoffanreicherung in der Biomasse. Dadurch verringert sich die Problematik einer nicht ausreichenden Sensitivität der Analytik, welche bei Messungen in der Wasserphase häufig auftritt. Geht es um Fragen der Herkunft von Stoffen oder um deren Verhalten in den Gewässern, helfen Biota-Untersuchungen wenig, da zur Erfassung von Transport- oder Umsetzungsprozessen Konzentrationen und Frachten dieser Stoffe auch in der Wasserphase benötigt werden. Allerdings helfen auch diese Untersuchungen nur, wenn eine ausreichend sensitive Analytik quantitative Ergebnisse (also Werte größer Nachweisgrenze ( $>\mathrm{NG}$ ) bzw. größer Bestimmungsgrenze (>BG)) liefert.

Die organischen Spurenstoffe der QZV Chemie OG wurden in der Wasserphase im Rahmen der nationalen Gewässerzustandsüberwachung im Neusiedler See selber bisher nicht unter- sucht. Von nationaler Seite wird die Überblicksmessstelle an der Wulka (Messstelle Seehof) vor der Mündung in den Schilfgürtel des Neusiedler Sees als Referenz für den See herangezogen, da die Wulka den dominanten oberflächlichen Zufluss für den See darstellt (Abb. 2). In Ungarn wurden im ungarischen Südteil des Sees in den Jahren 2005 und 2009 Untersuchungen einer Vielzahl der zu diesem Zeitpunkt geregelten organischen Spurenstoffe in der Wasserphase durchgeführt, und die Untersuchungsergebnisse liegen noch aus dem Projekt „Neusiedlersee - ökodynamische Rehabilitation" (Zessner et al. 2012) für die Bearbeitung dieses Artikels vor. Weitere Untersuchungen auf ungarischer Seite zur Überwachung von JD-UQN und ZHK-UQN werden z. B. derzeit im Rahmen des EU-Projektes REBEN (REBEN 2019) durchgeführt und standen für diese Auswertungen noch nicht zur Verfügung.

Neben den nationalen Messprogrammen liegen in Wulka, Wulkamündung und Neusiedler See Untersuchungen aus unterschiedlichen Forschungsprojekten vor, die im Laufe der letzten Jahre in Österreich durchgeführt wurden. Bei diesen Projekten standen nicht die Überwachung des Gewässerzustands und die Frage der Einhaltung von UQN im Vordergrund, sondern Überlegungen zu Herkunft und Verhalten von Stoffen. Bei der Auswahl der untersuchten Parameter ging es daher nicht um eine umfassende Betrachtung aller geregelten Stoffe, sondern um Stoffe, bei denen quantitative Analyseergebnisse in unterschiedlichen Bereichen $\mathrm{zu}$ erwarten sind, und die sich durch Herkunft (Abwasserentsorgung, Lufttransport, Landwirtschaft) und Umweltverhalten (Abbaubarkeit, Adsorptionseigenschaften) unterscheiden.

Im Projekt Neusiedler See - ökodynamische Rehabilitation - Betrachtungen zur Wasserqualität der Raab (Zessner et al. 2012) stand in Hinblick auf anthropogene Spurenstoffe die Frage im Vordergrund, wieweit diese sich im Falle eines Austrags von außen in der Wasserphase des Sees aufkonzentrieren können und welche Belastungen in Flüssen, die potenziell für eine Dotation in Frage kommen, vorliegen. Untersucht wurden die Parameter aus der QZV Chemie OG, 4-Nonylphenol techn., 4-Octylphenol, Alachlor, Anthracen, Atrazin, Bisphenol A, Di(2ethylexyl)phthalat, Dichlormethan, ED- 


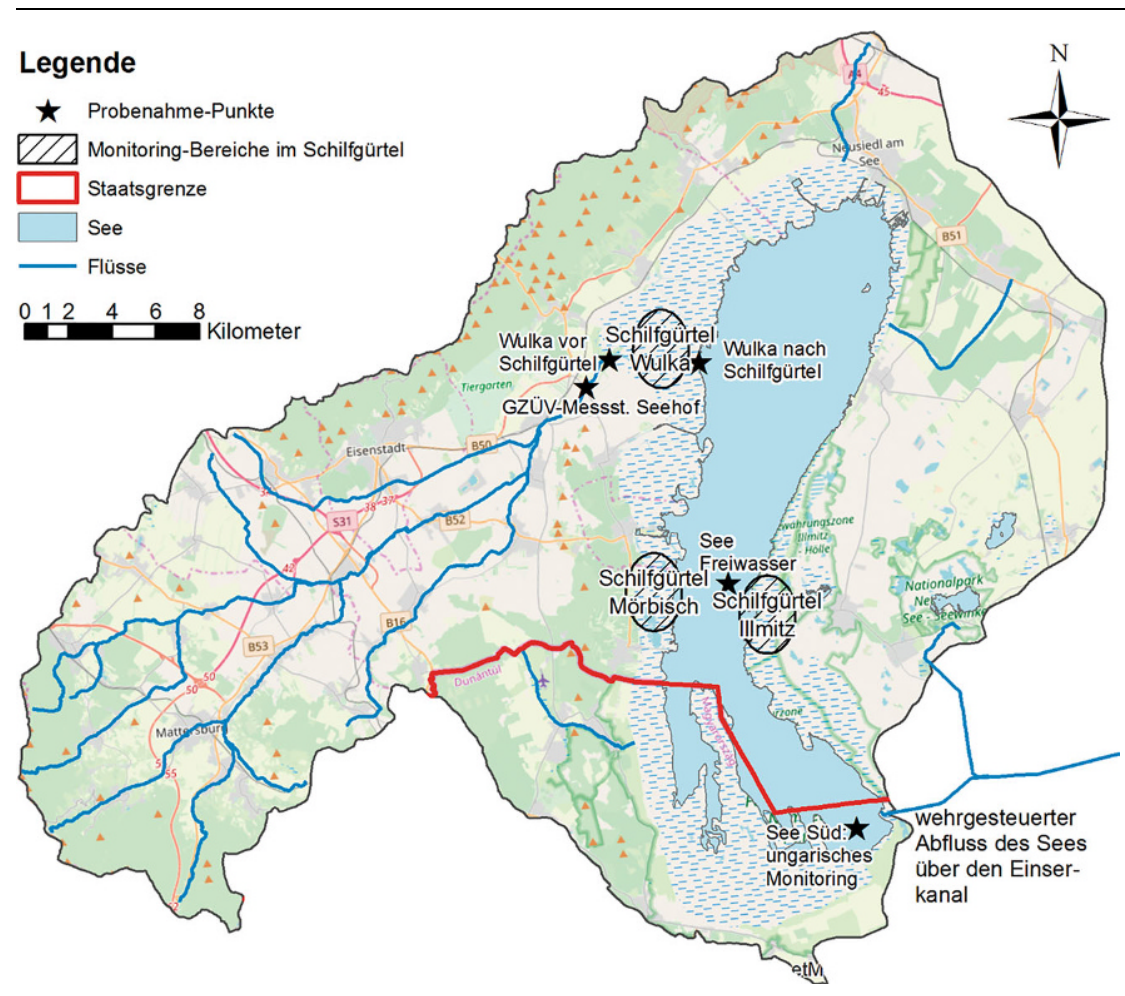

Abb. 2 Einzugsgebiet des Neusiedler Sees und Lage von Messstellen und beprobten Schilfbereichen. Datenquellen: Basemap: OpenStreetMap contributors, CC-BY_SA; Gewässernetz: Bundesberichtsgewässernetz V1 3 (2017) www.umweltbundesamt.at CC-BY3.0 AT

TA, Fluoranthen, LAS, Naphthalin, NTA, Sebuthylazin, Simazin, Trichlormethan und zusätzlich 1,5 Naphthalindisulfonat, 2,6-Naphthalindisulfonat, Carbamazepin, N,N-Dimethylsulfamid in der Wulka vor dem Schilfgürtel, in der Wulka nach dem Schilfgürtel und im Neusieder See selbst (siehe Abb. 2). Weitere Untersuchungen in Ikva, Rabnitz und Raab werden an dieser Stelle nicht weiter verwendet.

Das Projekt STOBIMO-Spurenstoffe (Kittlaus et al. 2019; Clara et al. 2019) fokussiert auf die Identifikation von Eintragspfaden von Spurenstoffen. Messungen von Konzentrationen in der Wasserphase und Schwebstoffen wurden in der Wulka sowie einer Reihe anderer Pilotgebiete mit dem Ziel durchgeführt, Gewässerfrachten zur Validierung von Ergebnissen einer Emissionsmodellierung zu ermitteln. Als Projektstoffe standen aus der QZV Chemie OG PBDE 6 , PFOS, Dibutylzinnverbindungen (DBT), Tributylzinnverbindungen (TBT) und die PAKs Benzo(a)pyren, Fluoranthen und Naphthalin sowie von den nicht geregelten Parametern Perfluoroctansäure (PFOA) im Fokus. Die Messstelle für diese Untersuchungen fenac, Acesulfam, Benzotriazol. Neben den Untersuchungen in der Wasserphase werden auch Sedimentproben und Proben aus dem Sediment-Porenwasser in den Bereichen des Schilfgürtels bei der Wulkamündung, bei Illmitz und bei Mörbisch auf eine Auswahl der oben genannten Stoffe hin untersucht.

\section{2 Überblick über den Belastungszustand}

In einem ersten Schritt soll der Belastungszustand des Neusiedler Sees mit anthropogenen Spurenstoffen über in der QZV Chemie OG geregelte Stoffe betrachtet werden. Dies ist zwar nur ein Aspekt der gesamten Problematik, allerdings jener, der im Falle einer Verfehlung von UQN mit direkten Handlungserfordernissen verknüpft ist.

Unter Anwendung der rechtlich verankerten Qualitätsziele für Wasser weist laut Nationalem Gewässerbewirtschaftungsplan NGP (BMLFUW 2017) nur ein sehr geringer Anteil der österreichischen Gewässer keinen guten $\mathrm{Zu}$ stand aufgrund der Belastung mit gemeinschaftlich oder national geregelten Schadstoffen (bzw. Spurenstoffen) auf. Anders ist die Situation bei bioakkumulierenden Stoffen, für die mit Richtlinie 2013/39/EU verstärkt sehr niedrige Umweltqualitätsziele in Biota festgelegt wurden. Hier weisen nationale Untersuchen von Biota sowie internationale Ergebnisse neben Quecksilber auch für die Summe der $\mathrm{PBDE}_{6}$ auf eine flächendeckende Überschreitung der UQN in Österreich und der EU hin. Dies spiegelt sich auch in den Ergebnissen der Fischuntersuchungen in Wulka und Neusiedler See aus Clara et al. (2015) wieder, wo für diese Stoffgruppe die UQN deutlich überschritten wird (Abb. 3). Aufgrund des ubiquitären Auftretens, einer Verteilung, die zu einem großen Teil über den Luftpfad erfolgt, und dem Eintrag über diffuse Pfade in die Gewässer ist dies jedoch kein Spezifikum für den Neusiedler See, sondern eine EU-weite Problematik, die langfristige und internationale Lösungskonzepte nötig macht.

Für die anderen untersuchten Parameter (Abb. 3) liegen die Messdaten entweder unter der Nachweisgrenze oder die UQN werden wie bei PFOS und den Toxizitätsäquivalenten für Dioxine und Furane (TEQ-PCDD/F/DLPCB) unterschritten. Speziell für PFOS ist anzumerken, dass von den Messwerten zur Biota-UQN nur ein gerin- 


\begin{tabular}{|c|c|c|c|c|c|c|}
\hline Spurenstoffe & & BG & NG & Biota-UQN & Wulka/Mündung & Neusiedler See \\
\hline & & & & & Aitel & Rotfeder \\
\hline HBCDD & $\mu \mathrm{g} / \mathrm{kg} \mathrm{FG}$ & 100 & 50 & 167 & $<N G$ & $<N G$ \\
\hline Heptachlor & $\mu \mathrm{g} / \mathrm{kg} \mathrm{FG}$ & 1,7 & 0,85 & \multirow{2}{*}{0,0067} & $<N G$ & $<N G$ \\
\hline Heptachlorepoxid & $\mu \mathrm{g} / \mathrm{kg} \mathrm{FG}$ & 1,7 & 0,85 & & $<N G$ & $<N G$ \\
\hline Hexachlorbutadien & $\mu \mathrm{g} / \mathrm{kg} \mathrm{FG}$ & 4 & 2 & 55 & $<N G$ & $<N G$ \\
\hline Dicofol & $\mu \mathrm{g} / \mathrm{kg} \mathrm{FG}$ & 4 & 2 & 33 & $<\mathrm{NG}$ & $<\mathrm{NG}$ \\
\hline Hexachlorbenzol & $\mu \mathrm{g} / \mathrm{kg} \mathrm{FG}$ & 4 & 2 & 10 & $<\mathrm{NG}$ & $<\mathrm{NG}$ \\
\hline PFOS & $\mu \mathrm{g} / \mathrm{kg} \mathrm{FG}$ & 2 & 1 & 9,1 & 7 & 8,1 \\
\hline Summe 6 PBDE & $\mu \mathrm{g} / \mathrm{kg} \mathrm{FG}$ & & & 0,0085 & 3,1 & 0,12 \\
\hline TEQ-PCDD/F/DL-PCB & $\mu \mathrm{g} / \mathrm{kg} \mathrm{FG}$ & & & 0,0065 & 0,002 & 0,00057 \\
\hline
\end{tabular}

Abb. 3 Ergebnisse des Fischmonitorings für organische Spurenstoffe in Wulka und Neusiedler See für Stoffe mit Biota-UQN (Daten

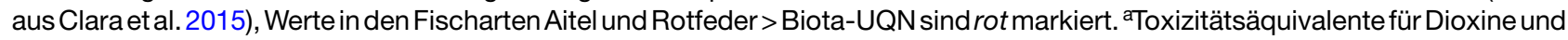
Furane

ger Spielraum besteht. Für die Summe aus Heptachlor und Heptachlorepoxid ist auffällig, dass die analytische Nachweisgrenze um einen Faktor von ca. 100 über der UQN liegt, wodurch eine Bewertung nicht möglich ist.

Wie der Name schon sagt, treten Spurenstoffe nur in Spurenkonzentrationen $(\mathrm{ng} / \mathrm{l}, \mu \mathrm{g} / \mathrm{l})$ in den Gewässern auf. Dies stellt eine große Herausforderung an die chemische Analytik dar, vor allem da für eine Umsetzung auf nationaler Ebene nur Standardanalytik mit entsprechend begrenzter Sensitivität angewendet werden kann. Betrachtet man die Ergebnisse des nationalen Gewässermonitorings entsprechend GZÜV in der Wulka, bei dem alle geregelten Stoffe 12 Mal untersucht wurden (PAK im Jahr 2013 und alle anderen Parameter bis auf jene, die im Fischmonitoring enthalten waren, im Jahr 2018), erkennt man, dass für die überwiegende Mehrzahl der Parameter durchwegs Werte unter der Nachweisgrenze (NG) oder der Bestimmungsgrenze (BG) ermittelt wurden. Bei 70 Parametern von organischen Spurenstoffen mit je 12 Analysen (840 Messwerte) waren lediglich 40 Messwerte $(4,8 \%)$ über der BG, davon 24 von EDTA und NTA, für die als einzige durchgehend quantifizierbare Analyseergebnisse ermittelt werden konnten. Für die meisten Parameter bedeuten diese Analyseergebnisse, dass eine deutliche Unterschreitung der JD-UQN bzw. ZHK-UQN in der Wulka gegeben ist.
Es gibt jedoch auch Parameter, bei denen die BG im Bereich oder über der JD-UQN liegt. Für diesen Fall wird in der QZV Chemie OG über Konvention geregelt, wie bei der Mittelwertbildung bzw. bei der Bewertung, ob der UQN überschritten ist, vorgegangen werden muss: „Messwerte, die unter der Bestimmungsgrenze liegen, sind auf die Hälfte des Werts der betreffenden Bestimmungsgrenze zu setzen." bzw. „Liegt ein arithmetischer Mittelwert unter der Bestimmungsgrenze, so wird dieser Wert als , unter der Bestimmungsgrenze' liegend bezeichnet. Für den Fall, dass die ... genannte JDUQN kleiner oder gleich der Bestimmungsgrenze ist, wird ein als ,unter der Bestimmungsgrenze' liegend bezeichneter arithmetischer Mittelwert nicht für die Beurteilung der Einhaltung der JD-UQN herangezogen." Damit kann dieser Mittelwert auch nicht zur Ausweisung eine Verfehlung des Qualitätsziels herangezogen werden.

In Abb. 4 sind Mittelwerte von Messergebnissen einer Auswahl national oder gemeinschaftlich geregelter Stoffe dargestellt. Neben den Ergebnissen der GZÜV sind in der Darstellung auch Ergebnisse anderer Messkampagnen an Wulka und vor allem auch vom Neusiedler See selber dargestellt. In die Darstellung aufgenommen wurden Stoffe, wo bei einem der Messprogramme Hinweise auf eine mögliche Grenzwertüberschreitung gegeben sind, das Problem mit Bestimmungsgrenzen oberhalb der JD-UQN gegeben ist oder weitgehend Messwerte über der BG erhoben werden konnten. Für die Auswertung wurde im Falle von Messwerten $<$ BG der Konvention der QZV Chemie OG gefolgt (siehe oben).

Für Nonylphenol und Octylphenol geben lediglich ungarische Untersuchungen aus dem Jahre 2005 Hinweise auf eine mögliche Zielverfehlung im Neusiedler See. Aktuellere Untersuchungen aus Österreich zeigen, dass die aktuelle Belastungssituation deutlich unter der JD-UQN liegt. Für NTA und EDTA liegt eine Vielzahl von Messwerten > BG vor, die zeigen, dass die JDUQN im Neusiedler See deutlich unterschritten wird. In der Wulka betragen die Messwerte für EDTA noch etwa 50\% der JD-UQN. Im See selber liegen die Messwerte jedoch deutlich niedriger als in der Wulka.

Bei den PAK Benzo(a)pyren und Fluoranthen tritt die Problematik auf, dass die Bestimmungsgrenze der Analytik im Bereich der JD-UQN liegt oder höher ist. Eine eindeutige Bewertung ist daher nicht möglich. Hinweise auf eine mögliche Zielwertverfehlung ergeben sich aus einzelnen Messwerten > BG, die dann bei der Mittelwertbildung zu Werten über der BG und teilweise auch über der JD-UQN führen (Abb. 4). Für diese beiden Stoffe liegt eine Problematik für die Beurteilung auch in der hohen Relevanz einer Adsorption an Schwebstoffe. Ein Transport erfolgt überwiegend partikelgebunden (Zoboli et al. 2019). Damit werden Monitoringergebnisse stark von der Erfassung von Schwebstoffen bei der Probenahme beeinflusst. Im Zuge der Projekte STOBIMO-Spu- 


\begin{tabular}{|c|c|c|c|c|c|c|c|}
\hline & Nonylphenol & Octylphenol & Benzo(a)pyren & NTA & EDTA & Fluoranthen & PFOS \\
\hline \multirow[t]{2}{*}{ JD-UQN } & 0,3 & 0,1 & 0,00017 & 50 & 50 & 0,0063 & 0,00065 \\
\hline & \multicolumn{7}{|c|}{ Wulka GZÜV Messstelle Seehof (2013/2018) } \\
\hline $\mathrm{n}>\mathrm{BG} / \mathrm{n}$ & $1 / 12$ & $0 / 12$ & $2 / 12$ & $12 / 12$ & $12 / 12$ & $0 / 12$ & 0 \\
\hline \multirow[t]{2}{*}{$\mu \mathrm{g} / \mathrm{l}$} & $<$ BG $(0,1)$ & $<B G(0,1)$ & 0,0048 & 2,3 & 29,7 & $<$ BG $(0,02)$ & n.b. \\
\hline & \multicolumn{7}{|c|}{ Wulka aus "Neusiedlersee - ökodynamische Rehabilitation (Zessner et al. 2012)" } \\
\hline $\mathrm{n}>\mathrm{BG} / \mathrm{n}$ & $3 / 3$ & $1 / 3$ & 0 & $3 / 3$ & $3 / 3$ & $1 / 3$ & 0 \\
\hline \multirow[t]{2}{*}{$\mu \mathrm{g} / \mathrm{l}$} & 0,07 & $<B G(0,02)$ & n.b. & 6,1 & 10,2 & 0,027 & n.b. \\
\hline & \multicolumn{7}{|c|}{ Wulka, Untersuchungen aus den Projekten STOBIMO Spurenstoffe und REBEN (laufend) } \\
\hline $\mathrm{n}>\mathrm{BG} / \mathrm{n}$ & $0 / 2$ & 0 & $0 / 8$ & $2 / 2$ & $2 / 2$ & $1 / 8$ & $9 / 9$ \\
\hline \multirow[t]{2}{*}{$\mu \mathrm{g} / \mathrm{l}$} & $<$ BG $(0,025)$ & n.b. & $<$ BG $(0,0002)$ & $<B G(0,5)$ & 26,5 & $<B G(0,002)$ & 0,0050 \\
\hline & \multicolumn{7}{|c|}{ Neusiedler See Süd (nationales Ungarischen Monitoring, 2005/2009) } \\
\hline $\mathrm{n}>\mathrm{BG} / \mathrm{n}$ & $8 / 8$ & $2 / 8$ & $1 / 9$ & 0 & 0 & $9 / 9$ & 0 \\
\hline \multirow[t]{2}{*}{$\mu \mathrm{g} / \mathrm{l}$} & 0,9 & 5 & $<\mathrm{BG}(0,001)$ & n.b. & n.b. & 0,0042 & n.b. \\
\hline & \multicolumn{7}{|c|}{ Neusiedler See aus "Neusiedlersee - ökodynamische Rehabilitation (Zessner et al. 2012)" } \\
\hline $\mathrm{n}>\mathrm{BG} / \mathrm{n}$ & $2 / 3$ & $0 / 3$ & 0 & $3 / 3$ & $3 / 3$ & $0 / 3$ & 0 \\
\hline \multirow[t]{2}{*}{$\mu \mathrm{g} / \mathrm{l}$} & 0,04 & $<$ BG $(0,01)$ & n.b. & 2,4 & 2,5 & $<B G(0,01)$ & n.b. \\
\hline & \multicolumn{7}{|c|}{ Neusiedler See, Untersuchungen aus dem Projekt REBEN (laufend) } \\
\hline $\mathrm{n}>\mathrm{BG} / \mathrm{n}$ & $0 / 2$ & 0 & $1 / 6$ & $0 / 2$ & $2 / 2$ & $1 / 6$ & $5 / 7$ \\
\hline$\mu \mathrm{g} / \mathrm{l}$ & $<\mathrm{BG}(0,025)$ & n.b. & 0,0002 & $<$ BG $(0,5)$ & 2,8 & 0,0038 & 0,0007 \\
\hline
\end{tabular}

Abb. 4 Mittlere KonzentrationenausgewählterorganischeSpurenstoffe derQZVChemieOGinWulkaund NeusiedlerSeeausunterschiedlichen Quellen, Mittelwerte > JD-UQN sind rotmarkiert. $n>B G$ Anzahl derAnalyseergebnisse > Bestimmungsgrenze; $n$ Anzahl der Analysen; n.b. nicht bestimmt

renstoffe (Clara et al. 2019) und REBEN (REBEN 2019) wurden gezielt Schwebstoffproben aus der Wulka bzw. Sedimentproben aus dem Schilfgürtel des Sees entnommen. Für Benzo(a)pyren wurden dabei im Mittel Konzentrationen von $85 \mathrm{ng} / \mathrm{gTM}$ im Schwebstoff und $20 \mathrm{ng} / \mathrm{gTM}$ im Sediment ermittelt, für Fluoranthen $150 \mathrm{ng} / \mathrm{gTM}$ bzw. $100 \mathrm{ng} / \mathrm{gTM}$ (siehe auch Tab. 1). Bereits ein Schwebstoffgehalt von $10 \mathrm{mgTM} / \mathrm{l}$ und weniger bei Benzo(a)pyren und 100mgTM/l Fluoranthen in einer Probe kann daher zu Messergebnissen>JDUQN führen. Ein Risiko für eine Überschreitung der UQN ist daher gegeben.

PFOS wird in wesentlich geringerem Ausmaß an Feststoffe adsorbiert und wird in Gewässern überwiegend in gelöster Form transportiert (Zoboli et al. 2019). Im Zuge der GZÜV wurde PFOS nicht in der wässrigen Phase, sondern lediglich in Biota gemessen. Dadurch liegen auch in der Wulka keine nationalen Messdaten vor. Durch Einsatz einer chemischen Analytik in den Projekten
STOBIMO-Spurenstoffe und REBEN mit einer hohen Sensitivität konnten für diesen Parameter Ergebnisse > BG ermittelt werden. In der Wulka wird die JD-UQN deutlich überschritten. Im Neusiedler See kommt es zu einer merklichen Abnahme der Konzentrationen. Die Messergebnisse liegen hier im Durchschnitt jedoch immer noch über der JD-UQN. Diese Ergebnisse zeigen ein geringfügig anderes Bild als die Messungen in Biota, wo die UQN knapp unterschritten wurde. Generell ist jedoch bekannt, dass die JD-UQN für PFOS ein etwas strengeres Schutzziel darstellt als die Biota-UQN (Clara et al. 2019). Da jedoch auch in Biota die UQN annähernd erreicht wird, zeigt sich, dass die PFOS-Belastung von Wulka und Neusiedler Sees als sensibel zu betrachten ist, auch wenn durch nationales Monitoring bisher keine Zielverfehlung nachgewiesen wurde.

Zusammenfassend lässt sich feststellen, dass für die überwiegende Zahl der national und gemeinschaftlich ge- regelten anthropogenen Spurenstoffe die Belastung in der Wulka keine Überschreitung der JD-UQN vorliegt und diese zumeist deutlich unterschritten wird. Dadurch ist für diese Stoffe eine Zielverfehlung im See unwahrscheinlich. Ganz auszuschließen ist ein gewisses Risiko einer Zielverfehlung auch für diese Stoffe jedoch nicht, da aufgrund der spezifischen Situation des Sees mit einer Verdunstung, die den Niederschlag übersteigt, auch eine Anreicherung von Stoffen in der Wasserphase möglich bzw. ein relevanter direkter Eintrag von Stoffen in den See z. B. über Deposition oder eine Mobilisierung aus Sedimenten nicht auszuschließen ist (siehe auch Kap. 4.) und eine umfassende Kontrolle aller Stoffe durch Monitoring im See nicht vorliegt.

Aus der Gruppe der anthropogenen Spurenstoffe ist für $\mathrm{PBDE}_{6}$ wie auch in ganz Österreich und in weiten Teilen der EU in Wulka und Neusiedler See eine Zielverfehlung gegeben. Die Problematik ist hier ähnlich wie bei 


\begin{tabular}{|c|c|c|c|c|c|c|c|c|}
\hline & $\begin{array}{l}\text { Wulka vor } \\
\text { Schilfgürtel }\end{array}$ & $\begin{array}{l}\text { Wulka nach } \\
\text { Schilfgürtel }\end{array}$ & $\begin{array}{l}\text { See Freiwas- } \\
\text { ser }\end{array}$ & $\begin{array}{l}\text { Illmitz/ } \\
\text { Mörbisch } \\
\text { Schilf-Wasser }\end{array}$ & $\begin{array}{l}\text { Illmitz/ } \\
\text { Mörbisch } \\
\text { Porenwasser } \\
\text { Sediment }\end{array}$ & $\begin{array}{l}\text { Wulka } \\
\text { Schwebstoffe }\end{array}$ & $\begin{array}{l}\text { Wulka Schilf-Se- } \\
\text { diment }\end{array}$ & $\begin{array}{l}\text { Illmitz/ } \\
\text { Mörbisch } \\
\text { Schilf-Sedi- } \\
\text { ment }\end{array}$ \\
\hline Einheit & $\mu g / L$ & & & & & $\mu \mathrm{g} / \mathrm{kg} \mathrm{TM}$ & & \\
\hline Stoff & \multicolumn{8}{|c|}{ Acesulfam - Süßungsmittel } \\
\hline$n / n>B G$ & $6 / 6$ & $3 / 3$ & $5 / 5$ & $11 / 11$ & $1 / 1$ & 0 & $2 / 0$ & $4 / 0$ \\
\hline MW & 0,310 & 0,710 & 0,500 & 0,550 & 0,002 & n.b. & $<5$ & $<5$ \\
\hline Stoff & \multicolumn{8}{|c|}{ Benzo(a)pyren - PAK } \\
\hline$n / n>B G$ & $12 / 2$ & $4 / 1$ & $5 / 2$ & $11 / 4$ & $2 / 2$ & $3 / 3$ & $3 / 2$ & $11 / 9$ \\
\hline MW & 0,0048 & $<0,0002$ & 0,0003 & 0,0006 & 0,002 & 85 & 14 & 20 \\
\hline Stoff & \multicolumn{8}{|c|}{ Benzotriazol - Industriechemikalie } \\
\hline$n / n>B G$ & $6 / 6$ & $1 / 1$ & $1 / 1$ & $3 / 3$ & 0 & 0 & 0 & 0 \\
\hline MW & 1,4 & 4,2 & 0,3 & 0,2 & n.b. & n.b. & n.b. & n.b. \\
\hline Stoff & \multicolumn{8}{|c|}{ Carbamazepin - Arzneimittel } \\
\hline$n / n>B G$ & $6 / 6$ & $3 / 3$ & $3 / 2$ & $9 / 5$ & 0 & 0 & $2 / 0$ & $4 / 0$ \\
\hline MW & 0,107 & 0,216 & 0,014 & 0,008 & n.b. & n.b. & $<5$ & $<5$ \\
\hline Stoff & \multicolumn{8}{|c|}{ Diclofenac - Arzneimittel } \\
\hline$n / n>B G$ & $6 / 6$ & $3 / 3$ & $3 / 1$ & $9 / 3$ & 0 & 0 & $2 / 0$ & $4 / 0$ \\
\hline MW & 0,331 & 0,760 & 0,009 & 0,007 & n.b. & n.b. & $<5$ & $<5$ \\
\hline Stoff & \multicolumn{8}{|c|}{ EDTA - Industriechemikalie } \\
\hline$n / n>B G$ & $12 / 12$ & $2 / 2$ & $2 / 2$ & $6 / 6$ & 0 & 0 & 0 & 0 \\
\hline MW & 29,7 & 26,5 & 2,8 & 1,7 & n.b. & n.b. & n.b. & n.b. \\
\hline Stoff & \multicolumn{8}{|c|}{ Fluoranthen - PAK } \\
\hline$n / n>B G$ & $12 / 0$ & $4 / 0$ & $5 / 1$ & $11 / 4$ & $2 / 2$ & $3 / 3$ & $3 / 3$ & $11 / 10$ \\
\hline MW & $<0,02$ & 0,002 & 0,004 & 0,0034 & 0,011 & 147 & 70 & 93 \\
\hline Stoff & \multicolumn{8}{|c|}{ N,N-Dimethylsulfamid - Pflanzenschutzmittel, Metabolit } \\
\hline$n / n>B G$ & $3 / 3$ & $5 / 5$ & $5 / 5$ & $6 / 6$ & 0 & 0 & 0 & 0 \\
\hline MW & 0,050 & 0,045 & 0,140 & 0,137 & n.b. & n.b. & n.b. & n.b. \\
\hline Stoff & \multicolumn{8}{|c|}{ NTA - Industriechemikalie } \\
\hline$n / n>\mathrm{BG}$ & $12 / 12$ & $2 / 0$ & $2 / 0$ & $6 / 0$ & 0 & 0 & 0 & 0 \\
\hline MW & 2,3 & $<0,5$ & $<0,5$ & $<0,5$ & n.b. & n.b. & n.b. & n.b. \\
\hline Stoff & \multicolumn{8}{|c|}{ PFOA - Industriechemikalie, Perfluortensid } \\
\hline$n / n>B G$ & $11 / 9$ & $5 / 5$ & $6 / 6$ & $13 / 13$ & $6 / 6$ & $3 / 0$ & $7 / 3$ & $19 / 7$ \\
\hline MW & 0,0034 & 0,0042 & 0,0092 & 0,0104 & 0,0371 & $<0,25$ & 0,10 & 0,18 \\
\hline Stoff & \multicolumn{8}{|c|}{ PFOS - Industriechemikalie, Perfluortensid } \\
\hline$n / n>B G$ & $10 / 10$ & $5 / 5$ & $6 / 4$ & $12 / 7$ & $6 / 6$ & $3 / 2$ & $7 / 3$ & $19 / 8$ \\
\hline MW & 0,0040 & 0,0042 & 0,0008 & 0,0010 & 0,0052 & 0,7 & 0,1 & 0,2 \\
\hline
\end{tabular}

Quecksilber, dass die Belastungen ubiquitär und vor allem über den Luftpfad erfolgen. Unter den geregelten Stoffen wurde PFOS als ein Stoff mit einer kritischen Belastung im See identifiziert. Nicht eindeutig ist derzeit die Bewertung für die PAK Benzo(a)pyren und Fluoranthen. Die BGs liegen vielfach höher als die JD-UQN. Einzelne Befunde deuten jedoch auf die Möglichkeit einer Zielverfehlung hin.

\section{3 Überlegungen zur Herkunft anthropogenen Spurenstoffen}

\subsection{Quellen und Eintragspfade}

Sollen die Emissionsfrachten von Stoffen in die Gewässer und damit die resultierenden Immissionskonzentrationen und -frachten in den Gewässern zielgerichtet gesenkt werden, ist eine quantitative Erfassung von Quellen und/oder Eintragspfaden der Stoffe erforderlich. Die Möglichkeit, die Wirksamkeit von Maßnahmen im Vorfeld deren Umsetzung abschätzen und vergleichen zu können, hängt in einem hohen Maße von dem vorhandenen Systemverständnis ab. Während z.B. bei den Nährstoffen ein hohes Maß an Systemverständnis vorhanden ist (z.B. Schilling et al. 2011), stehen wir diesbezüglich bei den anthropogenen Spurenstoffen erst am Anfang.

Im Jahre 2012 wurde auf EU-Ebene das Guidance-Dokument Nr. 28 „Preparation of Priority Substances Emissions Inventory" erarbeitet (European Commission 2012). Dieses liefert eine Grundlage zur Erstellung von Emissionsinventaren für ubiquitäre persistente, bioakkumulierende und toxische Stoffe (uPBT) und andere Spurenstoffe. 


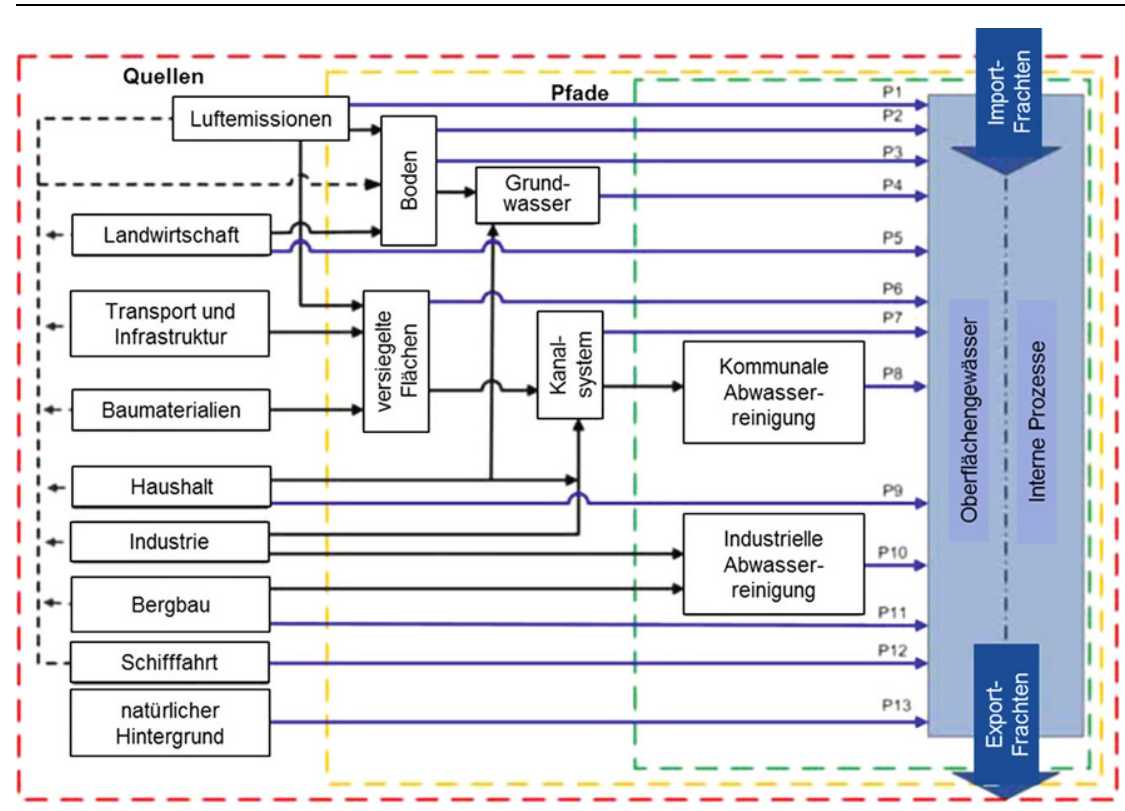

Abb. 5 Quellen und Eintragspfade von Stoffen und deren Erfassung in unterschiedlichen Ansätzen (Stufen) bei derErstellung vonEmissionsinventaren (modifiziertnach European Commission 2012). Abkürzungen: $P 1$ atmosphärische Deposition auf Gewässeroberflächen; $P 2$ Erosion; $P 3$ Oberflächenabfluss von unbefestigten Flächen; $P$ 4Zwischenabfluss, Drainagen und Grundwasser; $P 5$ Landwirtschaftlicher Direkteintrag; $P 6$ Oberflächenabfluss mit Regenwassereinleitungen aus Trennkanälen; $P 7$ Mischwasserüberlauf, Kanalisation ohne Reinigung; $P 8$ Gereinigtes kommunales Abwasser; $P 9$ Direkteinleitungen von Haushalten (ungereinigt bzw. gereinigt); P10 Gereinigtes industrielles Abwasser; P11 Direkte Einleitungen aus dem Bergbau; $P 12$ Direkte Einleitungen aus der Schifffahrt; $P 13$ Einträge aus dem natürlichen Hintergrund

Die Erstellung von Emissionsinventaren geschieht dabei auf Basis einer quantitativen Erfassung von Stoffeinträgen auf der Ebene von (Teil-)Einzugsgebieten. Für die Erstellung von Emissionsinventaren wird ein stufenweiser Ansatz vorgeschlagen (Abb. 5). Stufe 1 stellt ein Emissionsinventar aller Punktquellen dar. Stufe 2 (grün gestrichelte Umrandung in Abb. 5) ergänzt dieses durch Messungen von Immissionsfrachten und einer Abschätzung der über diffuse Pfade eingetragenen Stofffrachten aus Punkteinträgen und der Gewässerfracht. In Stufe 3 (gelb gestrichelte Umrandung in Abb. 5) werden die unterschiedlichen Eintragspfade (Kläranlagenabläufe, Mischwasserüberläufe, Emissionen aus Regenwasserkanälen, Erosion, Eintrag über das Grundwasser etc.) getrennt betrachtet. Für jeden Eintragspfad wird eine gesonderte Quantifizierung der Emissionsfrachten durchgeführt, aus diesen unter Berücksichtigung von Retention und $\mathrm{Ab}$ bau Immissionsfrachten errechnet und die berechneten Immissionsfrachten den beobachteten Emissionsfrachten gegenübergestellt. Stufe 4 geht noch einen Schritt weiter und verfolgt die Stoffe zurück zu ihren Herkunftsbereichen (source oriented). Der Anspruch dabei ist es, eine Stoffflussanalyse der Stoffe von deren Einsatzbereichen bis zu deren Gewässertransport zu erstellen. Diese Systembetrachtung kann in weiterer Folge dafür genutzt werden, um die Wirksamkeit sowohl von ,end of pipe“- als auch von „source control“Maßnahmen abschätzen zu können.

\subsection{Fallbeispiele}

Im vom BMNT geförderten Projekt STOBIMO-Spurenstoffe wurde der pfadorientierte Ansatz der Stufe 3 für Österreich auf einer Ebene von 754 Teileinzugsgebieten mithilfe des Emissionsmodells MoRE (MOdelling Regionalized Emissions, Fuchs et al. 2017) für eine Reihe von Spurenstoffen umgesetzt (Kittlaus et al. 2019; Clara et al. 2019). Die Wulka als der Hauptzubringer zum Neusiedler See wurde in Rahmen dieser Studie, unterteilt in 4 Teileinzugsgebiete, betrachtet und als Piloteinzugsgebiet in ein spezifisches Monitoringprogramm eingebunden (siehe auch Abschn. 2.1.). Im Folgenden wird auf die Emissionsmodellierung in diesem Projekt zurückgegriffen und es werden Ergebnisse für die Parameter PFOA, PFOS, Benzo(a)pyren und Fluoranten für das Wulkaeinzugsgebiet dargestellt. Zusätzlich wurden Depositonsmessungen von 5 Messstellen im Osten Österreichs herangezogen, um einen Eintrag dieser Stoffe direkt aus der Deposition auf den See und dessen Schilfgürtel abschätzen und den Einträgen über die Wulka gegenüber stellen zu können.

Abb. 6 zeigt deutlich die Unterschiede in der Herkunft zwischen PFOS und PFOA auf der einen Seite und Benzo(a)pyren und Fluoranthen auf der anderen Seite. Während bei den perfluorierten Tensiden (PFT) PFOS und PFOA der Eintrag über kommunale Kläranlagen überwiegt und auch Mischwasserentlastungen eine merkliche Rolle spielen, sind für die PAK Benzo(a)pyren und Fluoranthen diffuse Einträge aus der Fläche und hier vor allem über landwirtschaftliche Erosion dominant. Emissionsseitig könnte für die PFT daher über Maßnahmen bei den Kläranlagen eine Verringerung der Belastung der Wulka erreicht werden, während für die PAK der Schlüssel für eine Emissionsminderung beim Erosionsschutz liegen dürfte.

Bei den PFT und den PAK handelt es sich um uPBT-Stoffe, bei denen die Verbreitung über den Luftpfad eine Rolle spielt. Aus diesem Grund wurde aus Angaben zum Niederschlag als Komponente der Wasserbilanz des Sees (Wolfram et al. 2014) und Messungen von Konzentrationen im Niederschlag (Depositionsmessungen) aus dem Osten Österreichs im Rahmen von den von Clara et al. (2019) durchgeführten Untersuchungen $(n=5)$ eine Abschätzung des direkten Stoffeintrages über atmosphärische Deposition auf Schilfgürtel und See durchgeführt. Bereits die gemessenen Konzentrationen im Regenwasser weisen für die PFT mit $0,0013 \mu \mathrm{g} / \mathrm{l}$ für PFOS und $0,0020 \mu \mathrm{g} / \mathrm{l}$ für PFOA auf die Relevanz dieses Eintragspfades hin. Für PFOS wurde damit bereits im Niederschlag eine Konzentration über der JD-UQN für Oberflächengewässer gemessen. Die Messergebnisse für Benzo(a)pyren und Fluoranthen liegen zumeist unter der Bestimmungsgrenze und gingen mit 0,001 $\mu \mathrm{g} / \mathrm{l}$ in die Berechnung ein. Trotz der Unsicherheiten, die dieser Abschätzung aufgrund der begrenzten 


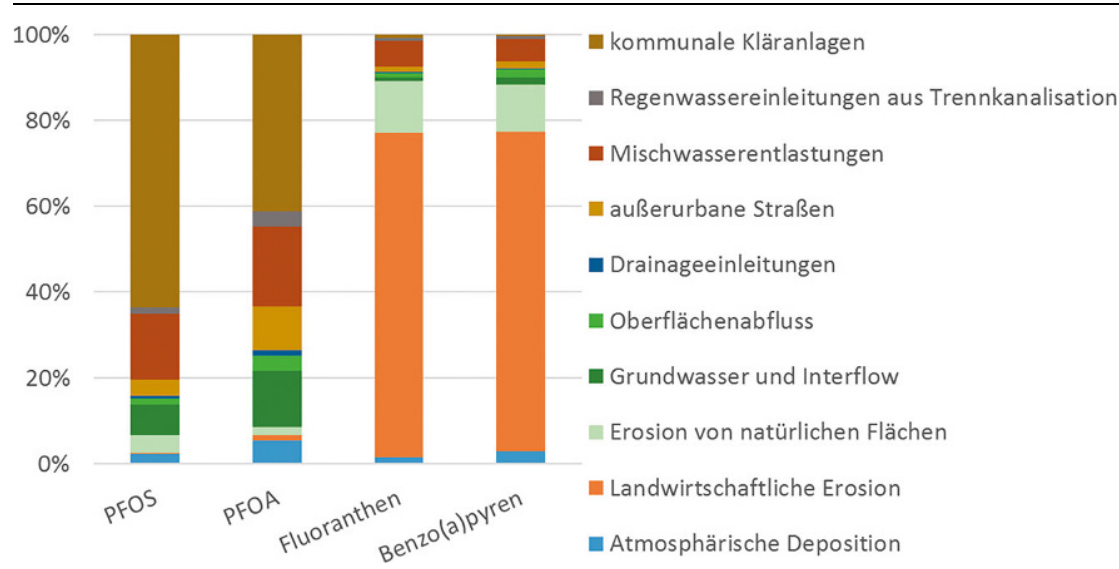

Abb. 6 Relative Bedeutung unterschiedlicher Eintragspfade für die Einträge von PFOS, PFOA, Fluoranthen und Benzo(a)pyren in die Wulka (basierend auf Clara et al. 2019)

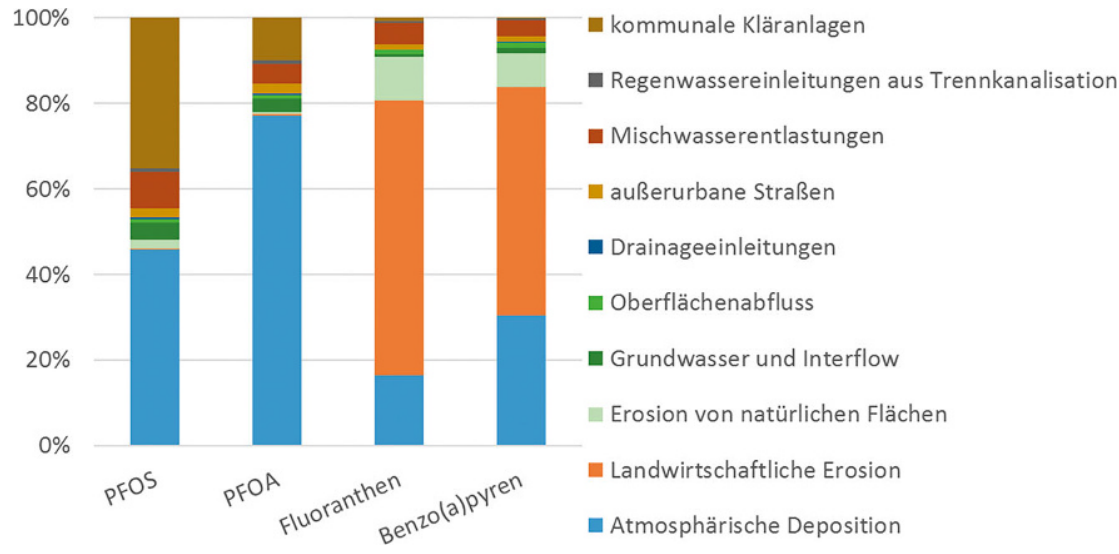

Abb. 7 Relative Bedeutung unterschiedlicher Eintragspfade für die Einträge von PFOS, PFOA, Fluoranthen und Benzo(a)pyren im Einzugsgebiet des Neusiedler Sees (inklusive Wulka)

Datenlage anhaften, verdeutlicht Abb. 7 die große Bedeutung, die der Eintrag über die Deposition auf die Gewässeroberfläche des Sees vor allem bei den PFT haben kann. Dies erschwert die Maßnahmensetzung zur Verringerung des Eintrages von PFT, da nur ein Teil der Einträge durch wasserwirtschaftliche Maßnahmen wie zusätzliche Reinigungsstufen bei der Abwasserreinigung reduziert werden kann. Hier wird sich weisen, welche Auswirkungen die bereits eingeleiteten Einschränkungen beim Einsatz von PFOS in unterschiedlichen Einsatzbereichen (z.B. Feuerlöscher, Imprägnierungen) in Zukunft haben werden. reguliert werden. Die anorganischen Stoffe bzw. chemischen Verbindungen Chlorid, Gesamtphosphor und Nitrat werden in Folge dazu verwendet, eine mögliches Umweltverhalten beispielhaft zu illustrieren, da für diese Stoffe im Gegensatz zu den anthropogenen Spurenstoffen langfristige und umfassende Datensätze zu den Konzentrationen in Wulka und See vorliegen.

In einer Chloridbilanz als Beispiel für einen persistenten, nicht adsorbierenden Stoff muss der Austrag den Eintrag über Zufluss und Deposition ausgleichen. In einer detaillierten Monatsbilanz zeigen Wolfram et al. (2007), dass beim Eintrag die Wulka die anderen Zuflüsse, aber auch die Deposition bei weitem übersteigt, und der Austrag in relevantem Ausmaß nur durch den Abfluss im Einserkanal erfolgt. Übernimmt man die Depositionsdaten aus Wolfram et al. (2007) und setzt man neben den Wasserbilanzkomponenten für den Zufluss die Konzentrationen in der Wulka (GZÜV Messstelle Seehof) und für den Abfluss über den Einserkanal die Konzentrationen im See (GZÜV Messstelle Donnerskirchen) an, so kann recht einfach eine langjährige Chloridbilanz für den See errechnet werden, die auch weitgehend ausgeglichen ist (Abb. 8b). Der Überhang des Wasserbilanzglieds Zufluss gegenüber dem Wasserbilanzglied Abfluss wird in der Chloridbilanz durch einen Überhang der Konzentration im Abfluss (=Konzentration im See) gegenüber der Konzentration im Zufluss (=Wulka) ausgeglichen.

Auch für persistente, nicht adsorbierende Spurenstoffe kann daher in ähnlicher Form eine Aufkonzentrierung im Seewasser gegenüber dem Zufluss durch die Wulka erwartet werden. Kann der Eintrag über die Deposition für einen Stoff vernachlässigt werden, wird der Anreicherungsfaktor (af) durch das Verhältnis Zufluss zu Abfluss bestimmt. Dies ist für Chlorid zu erkennen, wenn man die Zeitreihe 2000 bis 2018 der Konzentrationen in der Wulka (Messstelle Seehof) der Zeitreihe der Seemessstelle Donnerskirchen gegenüberstellt (Abb. 9). af liegt im Mittel dieser Jahre bei 3,2 und schwankt dabei getrieben vom Verhältnis von $\mathrm{Zu}$ - und Abfluss zum See zwischen etwa 3,9 im Jahr 2004 und 2,6 im Jahr 2011. Ein ähnlich hoher af ist ein deutliches Indiz für hohe Persistenz (geringer Abbau) eines Stoffes im See sowie eine geringe par- 


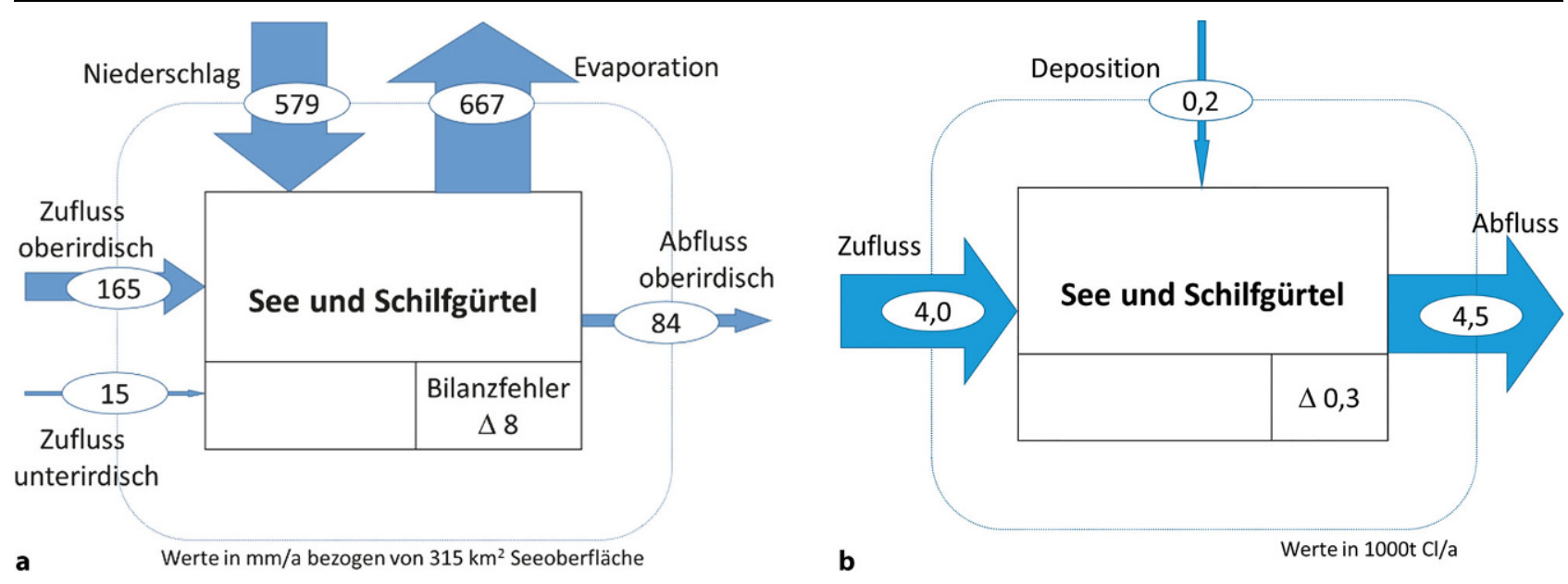

Abb. 8 a Mittlere Jahresbilanz des Neusiedler Sees für Wasser der Jahre 1965 bis 2018 (Datenquelle: DI Gerhard Kubu, Wien 2019 aus Sailer und Maracek 2019); b mittlere Jahresbilanz für Chlorid (eigene Berechnungen)

tikuläre Verlagerung in die Sedimente des Schilfgürtels.

Gesamtphosphor dient als Beispiel für einen Stoff, der in Wulka und See zu einem guten Teil partikulär gebunden vorliegt. Phosphor adsorbiert gut an Schwebstoffen, wird in anorganischer Form ausgefällt oder wird durch Algenwachstum in eine partikuläre Form übergeführt. Eine Deponierung von partikulär gebundenem Phosphor durch Sedimentation von Schwebstoffen im Bereich der Wulkamündung, aber auch in anderen Schilfbereichen ist gut dokumentiert und wird von Wolfram et al. (2019) behandelt. Diese partikuläre Ablagerung überprägt die Phosphorbilanz des Sees und führt dazu, dass im Vergleich zwischen Konzentrationen in der Wulka und im See eine deutliche Abreicherung stattfindet (Abb. 10). Der Anreicherungsfaktor oder in diesem Fall besser Abreicherungsfak-

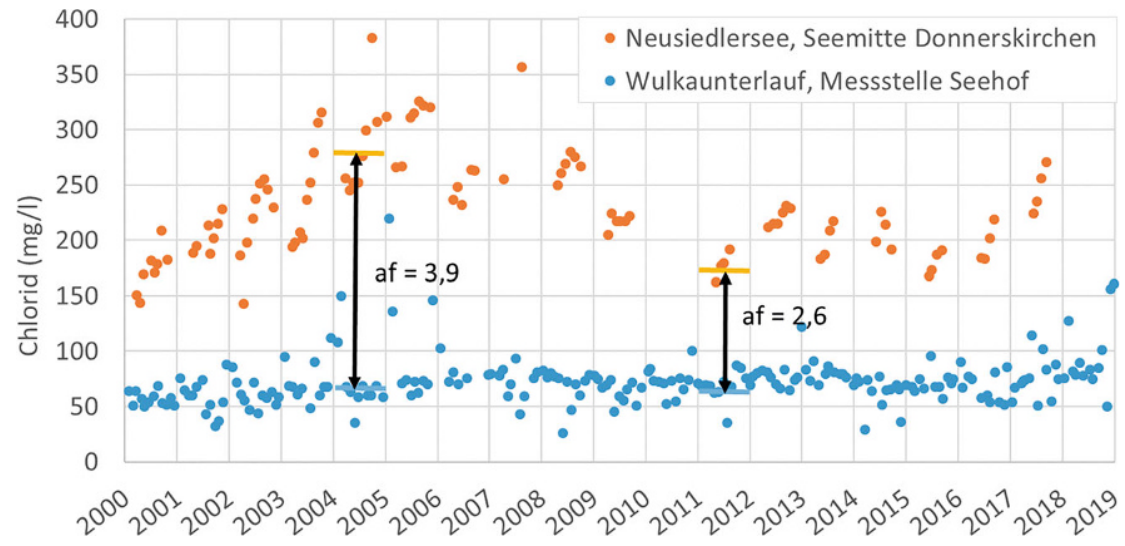

Abb. 9 Zeitreihen der Chloridkonzentrationen in der Wulka (GZÜV Messstelle Seehof) und Neusiedler See (GZÜV Messsstelle Donnerskirchen) tor (af) zwischen Wulka und See beträgt im Mittel der Jahre 2000 bis 20180,4 und schwankt zwischen $0,7 \mathrm{im}$ Jahr 2004 und 0,2 im Jahr 2014. Für persistente, gut adsorbierbare Spurenstoffe ist ein ähnliches Verhalten wie beim Phosphor zu erwarten, je nach Adsorbierbarkeit bzw. Verhältnis zwischen Vorkommen in gelöster Phase oder in partikelgebundener Form werden Stoffe über Schwebstoffe in die Schilfbereiche transportiert, dort durch Sedimentation abgelagert und so aus dem Freiwasser des Sees entfernt. Langfristig akkumulieren diese Stoffe im Sediment des Schilfgürtels des Sees und wie beim Phosphor (Wolfram et al. 2019) ist auch hier eine Mobilisierung und damit eine Rückführung eines Teiles der gespeicherten Stofffracht in das Freiwasser des Sees in Abhängigkeit von den Umgebungsbedingungen möglich. 


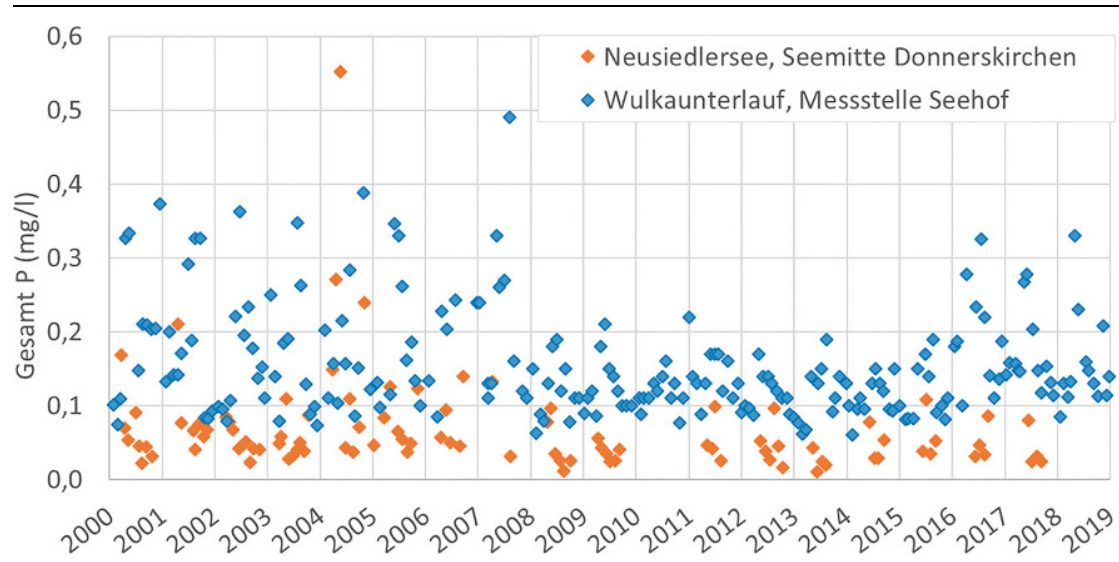

Abb. 10 Zeitreihen der Gesamtphosphorkonzentrationen in der Wulka (GZÜV Messstelle Seehof) und Neusiedler See (GZÜV Messstelle Donnerskirchen)

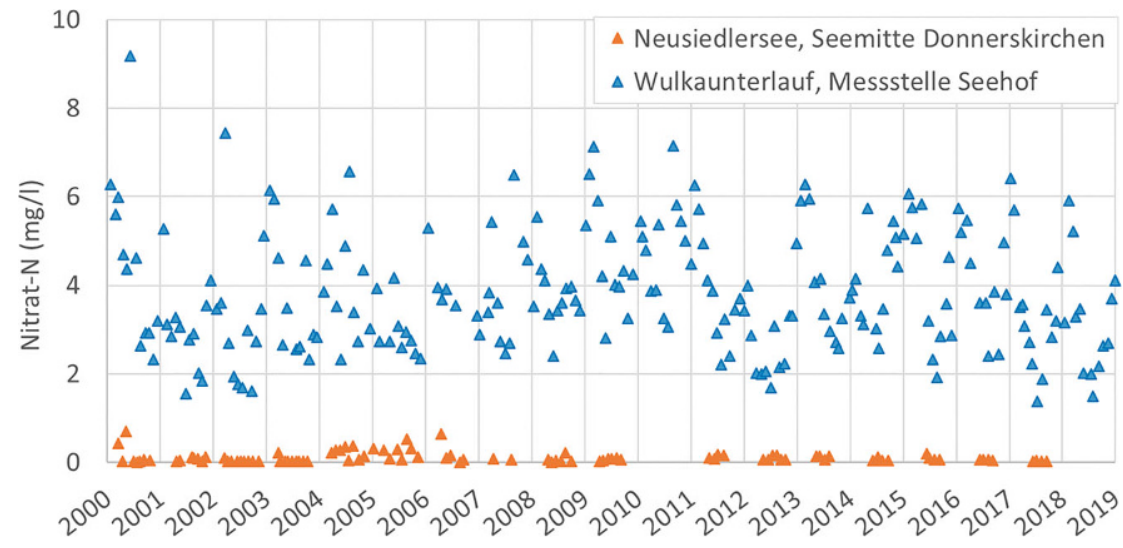

Abb. 11 Zeitreihen der Nitrat-Stickstoffkonzentrationen in der Wulka (GZÜV Messstelle Seehof) und Neusiedler See (GZÜV Messstelle Donnerskirchen)

ganischen Verbindungen, sogenannten Metaboliten. Selbst Stoffe, die grundsätzlich eine hohe Persistenz (schlechte Abbaubarkeit) aufweisen und z.B. in Kläranlagen nicht durch biologischen Abbau entfernt werden, können unter bestimmten Umweltbedingungen (lange Aufenthaltszeit, wechselnde aerobe/anaerobe Bedingungen, UV-Einstrahlung, pH-Wert) abgebaut oder zu anderen Verbindungen metabolisiert werden. Ein sehr geringer af kann hier vor allem für jene Stoffe, die keine hohe Adsorption an Feststoffe aufweisen, als Indikator gewertet werden, dass Ab- und Umbauprozesse in Schilfgürtel und See eine wesentliche Rolle für deren Umweltverhalten spielen.

\subsection{Fallbeispiele}

\subsubsection{Konzentrationsbetrachtungen}

In Tab. 1 ist eine Reihe von Analyseergebnissen von organischen Spuren- ten der errechnete Zahlenwert angegeben. Neben den Mittelwerten enthält die Tabelle auch die Anzahl der vorliegenden Messwerte sowie die Anzahl der Messungen $>$ BG. Für einige Parameter und Medien konnten nur einzelne oder keine Werte $>$ BG ermittelt werden. In diesen Fällen sind die quantitativen Aussagen mit einer großen Unsicherheit behaftet und können nur als erste Orientierung verstanden werden.

Die Tabelle zeigt zum einen Konzentrationswerte in der flüssigen Phase in der Wulka vor dem Schilfgürtel, in der Wulka nach dem Schilfgürtel, im Freiwasser des Sees, im Wasser des Schilfgürtels der Bereiche Illmitz und Mörbisch (siehe auch Abb. 2) und für einzelne Parameter zusätzlich im Porenwasser des Sediments der Schilfbereiche in Illmitz und Mörbisch. Zum anderen werden für einige Parameter auch Stoffkonzentrationen in Feststoffen der Schwebstoffe in der Wulka, des Sediments des Schilfbereiches bei der Wulkamündung und des Sediments der Schilfbereiche Illmitz und Mörbisch dargestellt.

Vergleicht man die Konzentrationen in der Wulka vor dem Schilfgürtel und im Freiwasser des Sees, fallen drei Parameter auf, bei denen der af deutlich über 1 liegt und es somit zu einer Anreicherung der Konzentrationen im See kommt: Acesulfam ( $a f=1,6), N, N$ Dimethylsulfamid $(a f=2,8)$ und PFOA $(a f=2,7)$. Bei diesen drei Stoffen ist aus den Analysedaten kein Hinweis auf eine Beeinflussung der Konzentrationen in der Wulka bei der Durchströmung des Schilfgürtels gegeben. Die Analyseergebnisse im Wasser der Schilfbereiche Illmitz und Mörbisch liegen in derselben Größenordnung wie im Freiwasser des Sees. Das heißt, eine weitere Anreicherung im Schilfgürtel ist nicht $\mathrm{zu}$ erkennen. Acesulfam wurde in den Sedimenten nicht gefunden und die Konzentration im Porenwasser ist sehr gering. Dies zeigt, dass eine Adsorption an Feststoffe und ein Austrag in das Schilfsediment nicht in relevantem Umfang stattfindet. Ein Umstand, der zu erwarten ist, da die Adsorptivität von Acesulfam sehr gering ist. Dasselbe gilt auch für N,N-Dimethylsulfamid. Hier wurde aus diesem Grund auf Analysen in Sediment und Porenwasser verzichtet. Etwas anders ist die Situation bei PFOA. Dieser Stoff ist auch in den Sedimenten nachweisbar und Konzentrationen im Porenwasser liegen deutlich über jenen in den an- 
deren Medien. PFOA wird daher auch aus dem Freiwasser über Feststoffe in das Sediment verfrachtet und es findet eine messbare Rücklösung aus dem Sediment in dessen Porenwasser statt. Für N,N-Dimethylsulfamid liegt af in etwa in jener Größe, wie sie einer theoretischen Anreicherung aufgrund des Überhangs der Verdunstung über den Niederschlag entsprechen würde. Dies spricht gegen einen Ab- oder Umbau, der im See stattfinden könnte, und für eine weitestgehende Persistenz dieses Stoffes auch unter den Umweltbedingungen des Neusiedler Sees. Etwas anders ist die Situation bei Acesulfam. Der af indiziert, dass hier Ab- oder Umbau-Prozesse stattfinden könnten, die $\mathrm{zu}$ einer Entfernung dieser Stoffe aus dem Freiwasser führen. Inwieweit dabei Metaboliten entstehen, kann beim derzeitigen Stand der Untersuchungen nicht gesagt werden. Für PFOA liegt der af im Bereich von N,N-Dimethylsulfamid. Allerdings wurde bereits in Abschn. 3.2. gezeigt, dass hier die Deposition auf den See eine wesentliche Rolle spielt. Für PFOA werden daher in Abschn. 4.2.2. auf Basis eines ersten Versuchs einer Stoffbilanzierung des Sees weitere Überlegungen durchgeführt.

Die weiteren betrachteten Stoffe weisen alle einen af von deutlich unter 1 auf: Benzo(a)pyren $(0,06)$, Benzotriazol $(0,18)$, Carbamazepin $(0,13)$, Diclofenac $(0,03)$, EDTA $(0,09)$, Fluoranthen $(0,40)$, NTA $(0,11)$ und PFOS $(0,21)$. Bei allen diesen Stoffen, außer bei den PAK Benzo(a)pyren und Fluoranthen sowie NTA gibt es keinen Hinweis auf eine Reduktion der Konzentrationen beim Durchströmen der Wulka durch den Schilfgürtel. Zudem kann für alle diese Stoffe mit Ausnahme von Benzo(a)pyren, Fluoranthen und PFOS entweder aufgrund von Messungen oder aufgrund theoretischer Überlegungen davon ausgegangen werden, dass eine Adsorption an Feststoffe und eine Verlagerung in das Sediment des Schilfgürtels über Sedimentation dieser Feststoffe keine wesentliche Rolle spielt. Für Benzotriazol, Carbamazepin, Diclofenac und EDTA kann daher geschlossen werden, dass sie trotz ihrer grundsätzlich hohen Persistenz unter den Umweltbedingungen des Neusiedler Sees einem relevanten biologischen und/oder photokatalytischen Ab- oder Umbau unterworfen sind, der zu einer deutlichen Reduktion der Konzentrationen im See führt. Ob der Endpunkt dieser Prozesse eine komplette Mineralisierung $\mathrm{zu} \mathrm{CO}_{2}$ ist, oder ob es zur Bildung von Metaboliten kommt, kann auf Basis der vorliegenden Untersuchungen nicht festgestellt werden.

Deutlich anders ist die Situation bei den PAK Benzo(a)pyren und Fluoranthen. Auch wenn hier aufgrund der hohen Anzahl an Befunden unter der Nachweisgrenze und der hohen Sensitivität der Analyseergebnisse gegenüber dem Gehalt an Feststoffen und somit dem Zeitpunkt der Probenahme (Hochwasserereignisse in der Wulka oder Windereignisse im See) eine hohe Unsicherheit in Hinblick auf quantitative Aussagen gegeben ist, können erste Hypothesen zum Verhalten dieser Stoffe im See aufgestellt werden. Beim Durchströmen der Wulka durch den Schilfbereich kommt es mit einer Sedimentation der Feststoffe auch zu einer relevanten Reduktion der PAK-Gehalte der Wulka. Die Schwebstoffe der Wulka sind im Vergleich zu den Sedimenten im Schilfbereich der Wulkamündung und den anderen untersuchten Schilfbereichen höher mit Benzo(a)pyren und Fluoranthen belastet. Gegenüber den Gehalten in der Wulka nach dem Schilfgürtel ist keine Abreicherung im Freiwasser des Sees zu erkennen. Die Werte deuten eher auf eine Erhöhung hin. Im Sediment der Schilfbereiche Illmitz und Wulka werden ebenfalls relevante PAK-Konzentrationen gefunden. PAKs werden mit den Feststoffen in den Schilfbereich transferiert und dort sedimentiert. Der Schilfgürtel fungiert daher als PAK-Speicher. Die Konzentrationen im Porenwasser des Schilfgürtels liegen im messbaren Bereich und deutlich über den Konzentrationen im See. Eine Mobilisation von PAK aus dem Sediment ist daher ein potenzieller Prozess für eine Rückbelastung des Sees. Quantitative Aussagen über dessen tatsächlicher Relevanz für die Belastung des Freiwassers des Sees können zurzeit jedoch nicht gemacht werden. Entsprechende weiterführende Untersuchungen sind im Rahmen des Projektes REBEN im Gange.

Auch die Ergebnisse für PFOS verdienen eine gesonderte Betrachtung. Der Transport über die Wulka erfolgt überwiegend in der gelösten Phase (Zoboli et al. 2019). Bei der Durchströmung der Wulka durch den Schilfgürtel kommt es zu keiner merklichen Konzentrationsveränderung. Im See selbst kommt es zu einer eindeutigen und deutlichen Reduktion der Konzentrationen.
Im Schilfsediment sind die PFOS-Konzentrationen fallweise messbar, im Vergleich mit Benzo(a)pyren und Fluoranthen sind die Konzentrationen allerdings sehr gering. Die Konzentrationen im Porenwasser des Schilfsediments liegen über den Konzentrationen im See, eine Mobilisierung aus dem Sediment dürfte daher in gewissem Umfang stattfinden. Gemeinsam mit PFOA sollen im Abschn. 4.2.2. über einen ersten Versuch von Stoffbilanzen weitere Überlegungen zu dem Verbleib von PFOS gemacht werden.

\subsubsection{Stoffbilanzen}

Für PFOS und PFOA liegt bei den organischen Spurenstoffen das umfangreichste Datenmaterial vor. Über Stoffbilanzen sollen nun wesentliche Stoffflüsse in und aus dem Neusiedler See einander gegenübergestellt werden. Die Zusammenstellung erhebt dabei keinen Anspruch auf quantitative Exaktheit, soll jedoch einen Vergleich von Größenordnungen und eine Identifikation von Bilanzlücken ermöglichen. Die Abschätzung nutzt die Wasserbilanzkomponenten für $\mathrm{Zu}$ - und Abfluss zum See und verknüpft diese mit PFOS- und PFOA-Konzentrationen in der Wulka und im Freiwasser des Sees, um den Eintrag über Zuflüsse bzw. den Austrag mit dem Abfluss über den Einserkanal zu berechnen. Zudem werden die in Abschn. 3.2. erläuterten Abschätzungen eines Eintrags über atmosphärische Deposition herangezogen, um auch diesen Eintragspfad in der Bilanz zu berücksichtigen. Wie Abb. 10a zeigt, kommt bei PFOS zu den Einträgen über die $\mathrm{Zu}$ flüsse ein Eintrag über die Deposition hinzu, der in derselben Größenordnung liegt. Der Austrag von PFOS über den Abfluss im Einserkanal ist aufgrund der geringen Konzentrationen im See im Vergleich mit den Einträgen sehr gering. So steht einem Eintrag von rund einem halben Kilo PFOS pro Jahr ein Austrag über den Abfluss von lediglich $0,01 \mathrm{~kg}$ pro Jahr gegenüber. Setzt man für die Konzentrationen in Feststoffen jene an, die im Schwebstoff der Wulka oder im Sediment des Sees gemessen wurden, und geht man von einer Verfrachtung von Feststoffen aus dem See in den Schilfgürtel von einer Größenordnung von 10.000 bis 20.000 tTM pro Jahr aus (vergl. Stalzer und Spatzierer 1987 in Wolfram et al. 2019), dann erkennt man, dass der Austragspfad aus dem See über eine Feststoffverlage- 


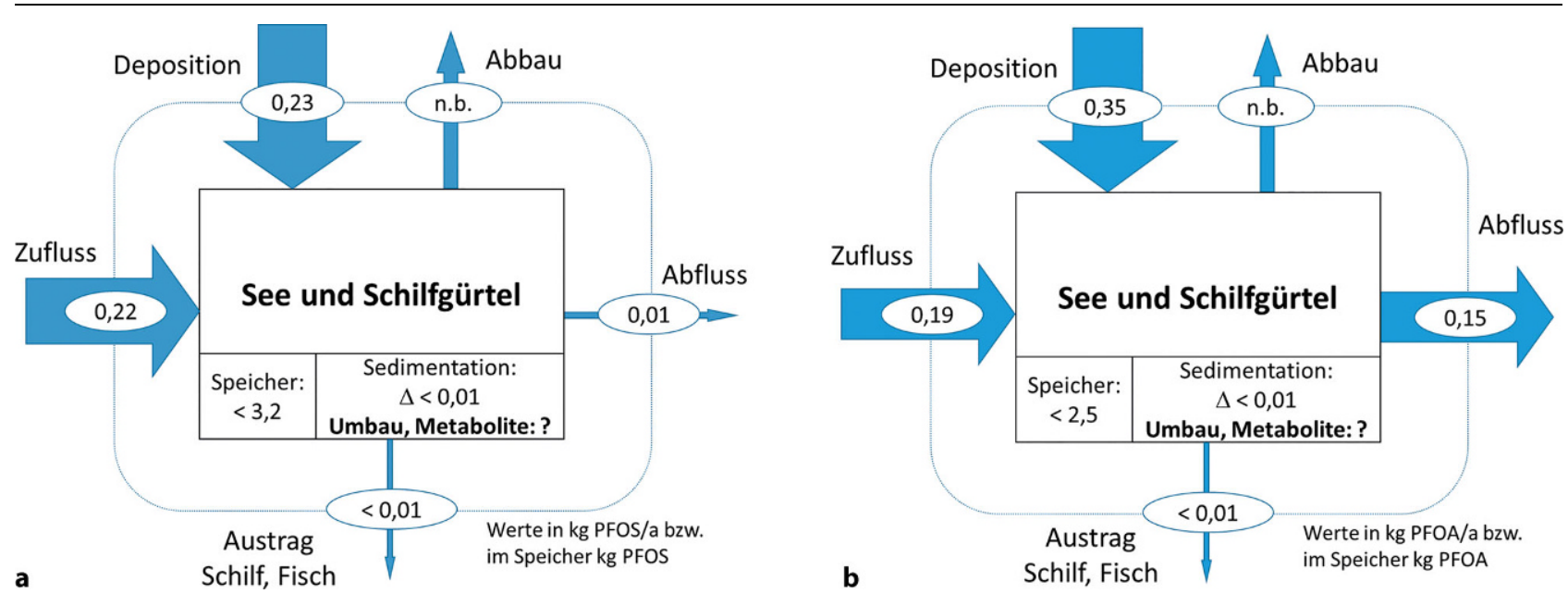

Abb. 12 Abschätzung von Jahresbilanzen des Neusiedler Sees für PFOS (a) und PFOA (b)

rung in den Schilfgürtel keine wesentliche Relevanz hat und maximal in der Größenordnung des oberirdischen Abflusses liegen kann. Ähnliches gilt für einen möglichen Austrag über Fischverzehr durch Wasservögel oder Fischfang. Selbst wenn man den unrealistischen Fall annimmt, dass die jährliche Fischentnahme dem Fischbestand von etwa $600 t$ (Angabe von Nemeth et al. 2003) entsprechen würde, so errechnet sich mit den PFOS-Konzentrationen in Biota (Abb. 3) ein Entzug über diesen Pfad, der deutlich unter $0,01 \mathrm{~kg}$ pro Jahr liegen würde.

Da auch ein relevanter Austrag von PFOS über Schilfschnitt von den Autoren dieser Arbeit als sehr unrealistisch eingeschätzt wird, lässt sich aus den bekannten Stoffflüssen der Überhang des Eintrags von PFOS über den Austrag nicht erklären. PFOS gilt als in der Umwelt extrem persistent (Beach et al. 2006). Unter gewissen Bedingungen erscheint eine Elimination von PFOS jedoch möglich. Dabei können allerdings überwiegend kürzerkettige PFT entstehen, deren Persistenz noch wesentlich höher ist als jene von PFOS (Trojanowicz et al. 2018). So indiziert auch die PFOS-Bilanz des Neusiedler Sees, dass PFOS weitgehend aus dem See eliminiert wird. Allerdings muss dies nicht eine komplette Mineralisation bedeuten. Wahrscheinlicher erscheint ein Umbau zu kurzkettigen PFT als Metaboliten, deren weiterer Verbleib in der Umwelt unbekannt ist. Weitere Untersuchungen sind im Rahmen des Projektes REBEN geplant, um dieser Frage nachzugehen.

Die Situation für PFOA ist ähnlich wie für PFOS (Abb. 12b). Der Eintrag über die Deposition dürfte jenen über die oberirdischen Zuflüsse übersteigen, und der bekannte Austrag kann den Verbleib der Einträge in den See nicht erklären. Unterschiedlich ist, dass die Elimination von PFOA wesentlich weniger weitgehend ist als bei PFOS, eine Seekonzentration verbleibt, die deutlich über jener in der Wulka liegt $(\mathrm{af}=2,7)$ und somit der Austrag über den Einserkanal in einer ähnlichen Größenordnung liegt wie der Eintrag über den Zufluss (überwiegend über die Wulka). Trotzdem ist zumindest jene Stofffracht, die über Deposition in den See eingetragen wird, nicht in den bekannten Austrägen wiederzufinden und auch hier indiziert die Bilanz relevante $\mathrm{Ab}$ - bzw. Umbauprozesse im See, als deren Endprodukt kurzkettige PFT als Metaboliten nicht auszuschließen sind.

\section{Schlussfolgerungen und Ausblick}

Wie bei Quecksilber als Vertreter der Metalle, liegt bei der Summe der PBDE, einer früher als Industriechemikalien (z.B. Flammschutzmittel) vielseitig eingesetzten Gruppe anthropogener Spurenstoffe, in Österreich und weiten Teilen der EU eine flächendeckende Überschreitung der UQN in Biota vor. Dies schlägt sich auch in der Belastungssituation des Neusiedler Sees nieder, ist aber kein Spezifikum des Sees und nur auf übergeordneter Ebene zu behandeln. Für die Summe aus Heptachlor und Heptachlorepoxid (Insektizid und dessen Metabolit) liegt die analytische Nachweisgrenze deutlich über der Biota-UQN und der JD-UQN. Messwerte liegen durchgehend unter der
Nachweisgrenze, und eine Bewertung der Belastungssituation ist nicht nur für den Neusiedler See nicht möglich.

Neben diesen auch auf Verwaltungsebene umfassend behandelten Problembereichen, weist PFOS aus der Gruppe der Perfluorierten Tenside (PFT), einer vielseitig (z. B. als Imprägnierungsmittel) eingesetzten Gruppe von Industriechemikalien, Konzentrationen in Wulka und Neusiedler See auf, die auf eine Überschreitung der JD-UQN hindeuten. Nicht eindeutig ist derzeit die Bewertung für die $\mathrm{zu}$ den Verbrennungsnebenprodukten gehörenden PAK Benzo(a)pyren und Fluoranthen. Die BGs liegen teilweise höher als die JD-UQN. Einzelne Befunde deuten jedoch auf die Möglichkeit einer Zielverfehlung hin. Da diese PAK überwiegend an Feststoffe gebunden transportiert werden, werden Analyseergebnisse stark von den bei der Probenahme erfassten Schwebstoffkonzentrationen bestimmt.

Bei den Emissionen in die Wulka dominieren bei den beiden PFT PFOS und PFOA Einträge über Kläranlagenabläufe. Auch Mischwasserentlastungen haben einen relevanten Anteil. Für die stark adsorbierenden PAK Benzo(a)pyren und Fluoranthen dominiert der Eintrag über landwirtschaftliche Erosion. Für den Neusiedler See kommen zu diesen Einträgen speziell bei den PFT noch relevante Einträge aus der atmosphärischen Deposition dazu, die in derselben Größenordnung wie die Einträge über die oberflächlichen Zuflüsse liegen.

Einmal in den See eingetragene Spurenstoffe können dort unterschiedlichem Verhalten unterworfen sein: (i) 
Anreicherung im Freiwasser des Sees durch den Überhang der Verdunstung über den Niederschlag; (ii) Adsorption an Feststoffe und eine Verlagerung in die Sedimente des Schilfgürtels gemeinsam mit Austrag und Sedimentation der Feststoffe, wodurch es zu einer Abreicherung im Freiwasser des Sees kommt; (iii) Ab- oder Umbau des Ausgangsstoffs unter den Umweltbedingungen des Sees, womit ebenfalls eine Abreicherung des Ausgangstoffs im Freiwasser gegeben ist. Allerdings kann es dabei zu einer Bildung von Metaboliten im See kommen. Daneben können auch Mischformen dieser Hauptarten des Umweltverhaltens auftreten.

Zu der Gruppe, bei der die Anreicherung im Freiwasser dominiert, ist nach derzeitigem Kenntnisstand der Pflanzenschutzmittel-Metabolit N,N-Dimethylsulfamid mit einem Anreicherungs-/ Abreicherungsfaktor (af) von 2,8 zu zählen. Auch das Süßungsmittel Acesulfam $(a f=1,6)$ geht in diese Richtung, könnte daneben aber auch einem begrenzten Ab- bzw. Umbau unterliegen. Die PAK Benzo(a)pyren $(a f=0,06)$ und Fluoranthen $(\mathrm{af}=0,40)$ gehören zu der Gruppe von Stoffen, bei denen eine Adsorption an Feststoffe und eine Verlagerung in das Sediment des Schilfgürtels im Vordergrund steht. Eine Rücklösung und Mobilisierung aus dem Sediment erscheint ein möglicher Belastungspfad dieser Stoffe für das Freiwasser des Sees $\mathrm{zu}$ sein. Das Korrosionsschutzmittel Benzotriazol $(\mathrm{af}=0,18)$, die Arzneimittelwirkstoffe Carbamazepin $(\mathrm{af}=0,13)$ und Diclofenac $(a f=0,03)$ sowie die Komplexbildner EDTA $(\mathrm{af}=0,09)$ und NTA $(a f=0,11)$ dürften zu einer Gruppe von Stoffen gehören, bei denen der Abbzw. Umbau überwiegt.

Die PFT PFOA $(\mathrm{af}=2,7)$ und PFOS $(\mathrm{af}=0,21)$ dürften eine Mittelstellung einnehmen, wo alle drei Verhaltensweisen zum Tragen kommen. Für PFOA kommt es zu einer deutlichen Anreicherung im Freiwasser des Sees. In messbarem Ausmaß wird PFOA aber auch in die Schilfsedimente verlagert und eine Bilanz des Sees unter Berücksichtigung der atmosphärischen Deposition indiziert eine hohe Relevanz von Abbau oder Umbau zu kurzkettigen PFT, über deren Verhalt im See noch keine In- formationen vorliegen. Bei PFOS findet ebenfalls eine messbare Verlagerung in das Sediment statt, die Ab- bzw. Umbauprozesse spielen hier jedoch eine noch wesentlich größere Rolle als bei PFOA, was bei einem ähnlichen Frachteintrag $\mathrm{zu}$ wesentlich geringeren Konzentrationen im Freiwasser und damit zu einer Abreicherung gegenüber den Konzentrationen in der Wulka führt.

Wie von Fenz und Clara (2019) festgestellt, erfordert Planung von Maßnahmen zur Reduktion der Gewässerbelastung mit anthropogenen Spurenstoffen einen stoffspezifischen und auch die charakteristischen Nutzungen spezifischer Einzugsgebiete berücksichtigenden Ansatz. Eine wirkungsvolle Verringerung der Gesamtbelastung der Gewässer ist nicht durch einzelne isolierte Maßnahmen zu erreichen, sondern durch Kombinationen von Maßnahmen, die den gesamten Lebenszyklus von Stoffen berücksichtigen. Maßnahmen zur Verringerung von Spurenstoffen in der aquatischen Umwelt können entweder beim Produzenten, dem Anwender der Produkte, bei der Abwasserentsorgung oder bei der Vermeidung von Einträgen aus der landwirtschaftlichen Erosion ansetzen.

PFOS werden sowohl über den Luftpfad als auch über den Wasserpfad in der Umwelt verteilt. Für diesen Stoff bestehen z. B. schon seit längerem starke Einschränkungen in Hinblick auf dessen Einsatzbereich (als Imprägnierungsmittel und in Feuerlöschern etc.). Diese Maßnahmen sind jedoch nicht immer umsetzbar und zeigen zudem oft erst nach langen Zeiträumen Wirkung. Auch ist gerade bei den PFT die Gefahr eines Umstiegs auf andere Formen, deren umwelttoxikologische Wirkungen ebenfalls bedenklich sind, zu beachten. Bei den PAK ist vor allem bei Emissionen aus Verbrennungsprozessen anzusetzen, die zu Belastungen von Boden und anderen Oberflächen führen. Dort, wo Vermeidungs- und Verminderungsstrategien nicht die erwünschte Wirkung zeigen, können Maßnahmen zur Vermeidung des Eintrags von Stoffen in die Gewässer ins Auge gefasst werden (je nach Herkunftsbereich z.B. weitergehende Abwasserreinigung für PFOS/ PFOA bzw. Arzneimittelwirkstoffe oder
Erosionsschutz in der Landwirtschaft für PAK).

Mit Bedachtnahme auf die große Zahl von in die aquatische Umwelt emittierten anthropogenen Spurenstoffen muss unabhängig von der Betrachtung von Einzelstoffen für den Neusiedlersee eine ganz besondere Vulnerabilität für diese Form der Belastung diagnostiziert werden. Dies liegt vor allem an der Funktion des Sees als Stoffsenke, an seiner geringen Tiefe, aber auch dem hohen Belastungsdruck über Siedlungstätigkeit und Landwirtschaft bei zugleich geringer mittlerer Abflusshöhe („Verdünnungspotenzial“), dem das Einzugsgebiet des Sees ausgesetzt ist. Spurenstoffe, die in den See eingetragen werden, können im Freiwasser aufkonzentriert, in den Sedimentbereichen des Schilfs gespeichert und von dort allenfalls wieder mobilisiert werden. Selbst wenn eine Vielzahl von Spurenstoffen durch Aboder Umbau aus dem Freiwasser eliminiert wird, ist über die Endprodukte eines solchen Umbaus noch wenig bekannt bzw. sind diese aufgrund der Vielzahl möglicher Stoffe nicht gänzlich zu überwachen. Um Fehlentwicklungen im See besser erkennen zu können, ist eine Intensivierung der Überwachung des Sees im Bereich der Spurenstoffe zu empfehlen, bei der eine Auswahl von Spurenstoffen und deren Metaboliten mit unterschiedlichen Herkunftsmustern und unterschiedlichem Umweltverhalten einer regelmäßigen Kontrolle über Sondermessprogramme unterzogen werden sollte.

Weitere Untersuchungen im Projekt REBEN werden sich auf die Erhöhung des Wissenstands in Hinblick auf die Rahmenbedingungen für eine Mobilisierung von PAK aus dem Sediment und auf eine mögliche Bildung von kurzkettigen Metaboliten beim Ab- bzw. Umbau von PFOS und PFOA fokussieren. Fallweise durchgeführte Projekte werden jedoch alleine nicht ausreichen, um eine qualitativ hochwertige Überwachung des Sees sicherzustellen.

Danksagung Die dargestellten Untersuchungen wurden mit den Mitteln des EU-Projekts ATHU53 REBEN „Angewandte hydrologische und limno- 
logische Basisuntersuchungen für das Projekt REBEN - Reed Belt Neusiedler See“ (INTERREG V-A AT-HU 2014-2020) gefördert. Unser Dank gilt auch dem BMNT für die Förderung des Projekts STOBIMO-Spurenstoffe zur Identifikation von Eintragspfaden von Spurenstoffen auf Ebene von Einzugsgebieten und für die Zurverfügungstellung der Daten aus dem GZÜV-Sondermonito- ring 2018 und dem Fischmonitoring von 2013

Funding Open access funding provided by TU Wien (TUW).

Open Access Dieser Artikel wird unter der Creative Commons Namensnennung 4.0 International Lizenz (http:// creativecommons.org/licenses/by/4. 0/deed.de) veröffentlicht, welche die Nutzung, Vervielfältigung, Bearbeitung, Verbreitung und Wiedergabe in jeglichem Medium und Format erlaubt, sofern Sie den/die ursprünglichen $\mathrm{Au}$ tor(en) und die Quelle ordnungsgemäß nennen, einen Link zur Creative Commons Lizenz beifügen und angeben, ob Änderungen vorgenommen wurden.

\section{Literatur}

Beach, S. A., Newsted, J. L., Coady, K., Giesy, J. P. (2006): Ecotoxicological Evaluation of Pefluorooctanesulfonate (PFOS). Rev Environ Contam Toxicol 186:133-174

BGBI. II Nr. 479/2006. Gewässerzustandsüberwachungsverordnung, Fassung vom 02.09.2019. Änderung: BGBl. II Nr. 465/2010, BGBl. II Nr. 363/2016, BGBl. II Nr. 128/2019

BGBl. II Nr. 96/2006. Verordnung des Bundesministers für Land- und Forstwirtschaft, Umwel und Wasserwirtschaft über die Festlegung des Zielzustandes für Oberflächengewässer, Qualitätszielverordnung Chemie Oberflächengewässer - QZV Chemie OG, Fassung vom 5.12.2016 (BGBl. II, Nr. 363/2016)

BMLFUW (2017): Nationaler Gewässerbewirtschaftungsplan 2015. Bundesministerium fü Land und Forstwirtschaft, Umwelt und Wasserwirtschaft. Wien, Juli 2017

Carvalho, R. N., Marinov, D., Loos, R., Napierska, D., Chirico, N., Lettieri, T. (2016): Monitoring-based Exercise: Second Review of the Priority Substances List under the Water Framework Directive. Joint Research Center JRC. JRC Science for Policy Report

CAS (2018): Information on number of CAS re gistrations, Chemical Abstracts Service, https:// www.cas.org/. Zugegriffen: 20.8.2019

Clara, M., Draxler, A. und Deutsch, K. (2015): Fisch-Untersuchungsprogramm 2013: GZÜV Untersuchungen. Bundesministerium für Landund Forstwirtschaft, Umwelt und Wasserwirtschaft. Wien, Juni 2015

Clara, M., Amann, A., Gabriel, O., Hochedlinger, G., Humer, M., Humer, F., Kittlaus, K., Kulcsar, S., Scheffknecht, C., Trautvetter, H., Zessner, M. und Zoboli, O. (2019): STOBIMO Spurenstoffe, Stoffbilanzmodellierung für Spurenstoffe auf Einzugsgebietsebene, im Auftrag des Bundesministeriums für Nachhaltigkeit und Tourismus, Endbericht in Vorbereitung

EU (2000): Richtlinie 2000/60/EG des Europäischen Parlaments und des Rates vom 23 Oktober 2000 zur Schaffung eines Ordnungsrahmens für Maßnahmen der Gemeinschaft im Bereich der Wasserpolitik. Amtsblatt Nr. L 327 vom 22/12/2000 S. $0001-0073$

EU (2006): Verordnung 1907/2006/EG des europäischen Parlaments und des Rates vom 18 Dezember 2006 zur Registrierung, Bewertung, Zulassung und Beschränkung chemischer Stoffe (REACH).

EU (2008/2013): Richtlinie 2008/105/EG des Europäischen Parlaments und des Rates übe Umweltqualitätsnormen im Bereich der Wasserpolitik vom 16. Dezember 2008 und Richtlinie 2013/39/EU des Europäischen Parlaments und des Rates vom 12. August 2013 zur Änderung der Richtlinien 2000/60/EG und 2008/105/EG in Bezug auf prioritäre Stoffe im Bereich de Wasserpolitik am 13. September 2013

European Commission (2012): Common Implementation Strategy for the Water Framework
Directive (2000/60/EG), Guidance document No. 28: Technical Guidance on the Preparation of an Inventory of Emissions, Discharges and Losses of Priority and Priority Hazardous Substances. European Commission.

Fenz, R. und Clara, M. (2019): Entwicklun gen auf EU Ebene. Wiener Mitteilungen (2019): Band 249, 1-18. Institut für Wassergüte/TUWien

Fuchs, S., Kaiser, M., Kiemle, L., Kittlaus, S. Rothvoß, S., Toshovski, S. et al. (2017): Modeling of Regionalized Emissions (MoRE) into Water Bodies. An Open-Source River Basin Management System. In: Water 9/4:239-248. DOI: $10.3390 /$ w9040239.

Van Gils, J. et al. (2019): The European Collaborative Project SOLUTIONS developed models to provide diagnostic and prognostic capacity and fill data gaps for chemicals of emerging concern. Environmental Sciences Europe, accepted Jirsa, F., Pirker, D., Krachler, R. und Bernhard Keppler. B. K. (2014): Total Mercury in Sediments, Macrophytes, and Fish from a Shallow Steppe Lake in Eastern Austria. Chemistry \& Biodiversity Vol. 11:1262-1275

Kittlaus, S., Gabriel, O., Clara, M., Scheffknecht, C., Zoboli, O., Hochedlinger, G., Saracevic, E., Humer, M., Zessner, M. (2019): Emissionsmodellierung organischer und anorganischer Spurenstoffe in Einzugsgebieten. Wiener Mitteilungen (2019) Band 249:169-196

Nemeth, E., Wolfram, G., Grubbauer, P., Rössler, M., Schuster, A., Mikschi, E. and Herzig, A. (2003): Interaction between fish and colonial wading birds within the reed beds of lake Neusiedl, Austria. In: Interactions beween Fish and Birds: Implications of Management, $\mathrm{Hg}$. I.G. Cowx, Blackwell Science Ltd, pp 139-150 ÖWAV (2013): ÖWAV-Positionspapier: Anthropogene Spurenstoffe in der aquatischen Umwelt. Österreichischer Wasser- und Abfallwirtschaftsverband

REBEN (2019): Angewandte hydrologische und limnologische Basisuntersuchungen für das Projekt REBEN - Reed Belt Neusiedler See. EUProjekt ATHU53 REBEN (INTERREG V-A AT-HU 2014-2020)

Sailer, C. L und Maracek, K. (2019): Der Neusiedler See - ein Überblick. Österreichische Wasser- und Abfallwirtschaft, 71/11-12, in diesem Heft

Schilling, C., Zessner, M., Kovacs, A., Hochedlinger, G., Windhofer, G., Gabriel, O., Thaler, S. Parajka, J., Natho (2011): Stickstoff- und Phosphorbelastungen der Fließgewässer Österreichs und Möglichkeiten zu deren Reduktion. Österreichische Wasser- und Abfallwirtschaft, 63/56:105-116

Slobotnik, J. and von der Ohe, P. C. (2015): Identification of the Danube River Basin Specific Pollutants and Their Retrospective Risk Assessment. in Hbd Env Chem 2015/39: 95-110. DOI
10.1007/698_2015_378, Springer-Verlag Berlin Heidelberg

Stalzer, W. und Spatzierer, G. (1987): Zusammenhang zwischen Feststoff- und Nährstoffbelastung des Neusiedler Sees durch Sedimentverfrachtung. Wissenschaftliche Arbeiten aus dem Burgenland 77: 93-226

Trojanowicz, M., Bojanowska-Czajka, A., Bartosiewicz, I., Kulisa, K. (2018): Advanced Oxidation/Reduction Processes treatment for aqueous perfluorooctanoate (PFOA) and perfluorooctanesulfonate (PFOS) - A review of recent advances. Chemical Engineering Journal, Volume 336:170-199

UBA (2018): Empfehlungen zur Reduzierung von Mikroverunreinigungen in den Gewässern. Hintergrundpapier, Umweltbundesamt, Dessau-Roßlau, April 2018

Wolfram, G. und Herzig, A. (2013): Nährstoffbilanz Neusiedler See. Wiener Mitteilungen 228:317-338

Wolfram, G., Deri, L. und Zech, S. [Red.] (2014): Strategiestudie Neusiedler See - Phase 1. Studie im Auftrag der Österreichisch-Ungarischen Gewässerkommission. Wien - Szombathely, pp 244 Wolfram, G., Donabaum, K. und Hintermaier, S. (2007): Stoffbilanz Neusiedler See 1992-2005 Studie i.A. d. Arbeitsgemeinschaft Natürliche Ressourcen (AGN), Wien, 106 pp.

Wolfram, G., Hainz, R., Hintermaier, S., Kum, G., Riedler, P., Zessner, M., Zoboli, O. und Herzig, A. (2019): Eintragspfade, Umsetzungsprozesse und Langzeitveränderungen von Nährstoffen im Neusiedler See. Österreichische Wasserund Abfallwirtschaft, 71/11-12, in diesem Heft Zessner, M., Gabriel, O., Schilling, K., Pannonhalmi, M., Sutheo, L., Kovács, M., Toth, I., Clement, A., Karches, T., Szilagyi, F., Kramer, T., Jozsa, J., Wolfram, G., Ruzicska, K. und Hintermaier, S. (2012): Neusiedler See - Ökodynamische Rehabilitation. Betrachtungen zur Wasserqualität der Raab. Studie i. A. des Amts de Burgenländischen Landesregierung, Abteilung 9 - Wasser und Abfallwirtschaft, Wien, Budapest, Györ, 189 pp.

Zoboli, O., Clara, M., Gabriel, O., Scheffknecht, Ch., Humer, M., Brielmann, H., Kulcsar, S. Trautvetter, H., Kittlaus, K., Amann, A., Saracevic, E., Krampe, J. and Zessner, M. (2019): Occurrence and levels of micropollutants across environmental and engineered compartments in Austria. Journal of Environmental Management 232:636-653. https://doi.org/10.1016/j jenvman.2018.10.074.

Hinweis des Verlags Der Verlag bleibt in Hinblick auf geografische Zuordnungen und Gebietsbezeichnungen in veröffentlichten Karten und Institutsadressen neutral. 